This report was prepared as an account of work sponsored by an agency of the United States Government. Neither the United States Government nor any agency thereof, nor any of their employees, makes any warranty, express or implied, or assumes any legal liability or responsibility for the accuracy, completeness, or usefulness of any information, apparatus, product, or process disclosed, or represents that its use would not infringe privately owned rights. Reference herein to any specific commercial product, process, or service by trade name, trademark, manufacturer, or otherwise does not necessarily constitute or imply its endorsement, recommendation, or favoring by the United States Government or any agency thereof. The views and opinions of authors expressed herein do not necessarily state or reflect those of the United States Government or any agency thereof.

\title{
Savannah River Site Computing Architecture (U)
}

WSRC-IM-91-18-1

February 26, 1997, Nitach g9, 1951

Westinghouse Savannah River Company

P. O. Box 616

Aiken, SC 29802 


\section{DRAFT}

\section{Savannah River Site Computing Architecture (U)}

WSRC-IM-91-18-1

February 26, 1991

Westinghouse Savannah River Company

P. O. Box 616

Aiken, SC 29802 


\section{Executive Summary}

A computing architecture is a framework for making decisions about the implementation of computer technology and the supporting infrastructure. Because of the size, diversity, and amount of resources dedicated to computing at the Savannah River Site (SRS), there must be an overall strategic plan that can be followed by the thousands of site personnel who make decisions daily that directly affect the SRS coinputing environment and impact the site's production and business systems. This plan must address the following requirements:

- There must be SRS-wide standards for procurement or development of computing systems (hardware and software). New programmatic directions are increasingly requiring that previously isolated computer systems and applications be joined together in cooperative information systems. Without standards, this necessary integration of systems with each other is, at best, difficult and, at worst, impossible because of technological and product differences. Choosing and using site standard computing products is also a way of reducing costs, as support infrastructure is required for fewer products.

- The site computing organirations must develop systems that end users find easy to use. Users today are rarely limited to a single computer-based system. Each user must learn how to manipulate multiple systems, which are often quite different in the interface they present to the user. A movement to standards in computer systems interfaces will greatly improve the usability, and hence the usefulness of our systems.

- Systems must be put in place to support the pirimary function of site information workers. Information workers (exempt, clerical, and a surprising number of production personnel) find that, in spite of the continuing drive to computerize data, it is increasingly difficult for them to locate the information they need to perform their jobs. What is needed is a site-wide, centrally administered "repository" of information describing site data. This data is in many different formats including computerized databases, documents and other text-based information, drawings, and image. This situation was quantified in a study of site personnel in 1985 (Reference 1) and since then there have been large increases in both personnel and data requirements at the site.

The developers of computer systems must be given tools that automate and speed up the development of information systems and applications based on computer technolosy. Many programming groups on site have large backlogs of applications which are not being developed for lack of available manpower. A large percentage of that manpower is devoted to maintaining existing applications and attempting to integrate systems and data from a variety of sources together.

This document describes a proposal for a site-wide computing architecture that addresses the above requirements. In summary, this architecture is standards-based, data-driven, and workstation-ariented with larger systerns being witlized for the delivery of needed information to users in a client-server relationship. 
A goal for SRS is to have one comprehensive computing architecture that is relevant for such diverse areas of computing as systems for management control, design and engineering, research and development, operational support, process control, office automation, and management information systems. This is an very ambitious undertaking, as these areas affect virtually all aspects of work at the site. This initial effort at defining the site architecture is focused on areas most applicable to traditional computer information systems. However, the architectural principles and directions put forth in this document are considered applicable to all areas of computing. Future development of the Computer Architecture, under the leadership of the site Computing Architecture and Standards Team (CAST) and involving those most knowledgeable in these areas, will expand the architecture to address any unique requirements of scientific and engineering computing, process computing, and classified computing applications.

The changes being proposed here are as much organizational and cultural as they are technological. This architecture proposal has five main points:

An SRS Computing Council will be established to set the gite's policy on computer matters. The creation of this Council was an element of the WSRC proposal to DOE to operate the site. This document assumes that this Council will be created and will sponsor the coordinating organizations described below.

- The site computing systems must be besed on a set of site standards for the development, purchase, and implementation of information systems, computer technology, and their supporting infrastructure. The major emphasis of these standards will be on the interfaces required for information systems integration. Standards will be based wherever possible on open national and international standards, although standardization on dominant proprietary standards and on specific products will be required to reduce the total number of computer products supported at the site to a more manageable set and to achieve the efficiencies possible with today's technology. This document calls for the establishment of a' site-wide Computing Architecture and Standards Team (CAST) to develop and manage these standards.

- Site data will be uniformly managed. A high-level site-wide organization is required to establish policy concerning the collection, storage, and dissemination of diata. Strategic site data must be organized into a model that describes SRS data entities and their relationships. This model will be implemented in a repository, which is a database about databases.

- The user of computer systems utiliving an intelligent wortsstation or personal computer participating in a site-wide computer network will become the central focus of the computing architecture. This is not to say that large mainframe computers and minicornputers will be obsoleted in the future, but that larger computer systems will provide various computing and information services which the end user will access via the personal workstation.

- There will be fundamental changes in the ways computer applications are developed and implemented on the gite. A major goal will be attaining the proper mix of buying generic application software versus building custom applications. This will require a cost/benefit analysis of how much site operating and business 
processes can be changed so as to utilize purchased software applications. C'ustom software development will be reserved for those cases where purchasing software solutions is not practical.

When custom software must be created, developers will utilize the power of the workstation and new computer-based tools that allow much more rapid application development. These tools are based on emerging technologies:

- CASE (computer aided software engineering)

- integrated data repository

- modular programming techniques and object oriented programming Systems (COPS)

- $\quad$ graphice' user interface (GUI)

- client-server computing

Figure 1 is a pictorial summary of the new architecture. The four major functional areas of computer technology, the platforms (computer hardware and operating systems), communications (networks), data, and applications are joined together via standards, data management, the power of the intelligent workstation, and the new technologies for application development. 


\section{Applications}

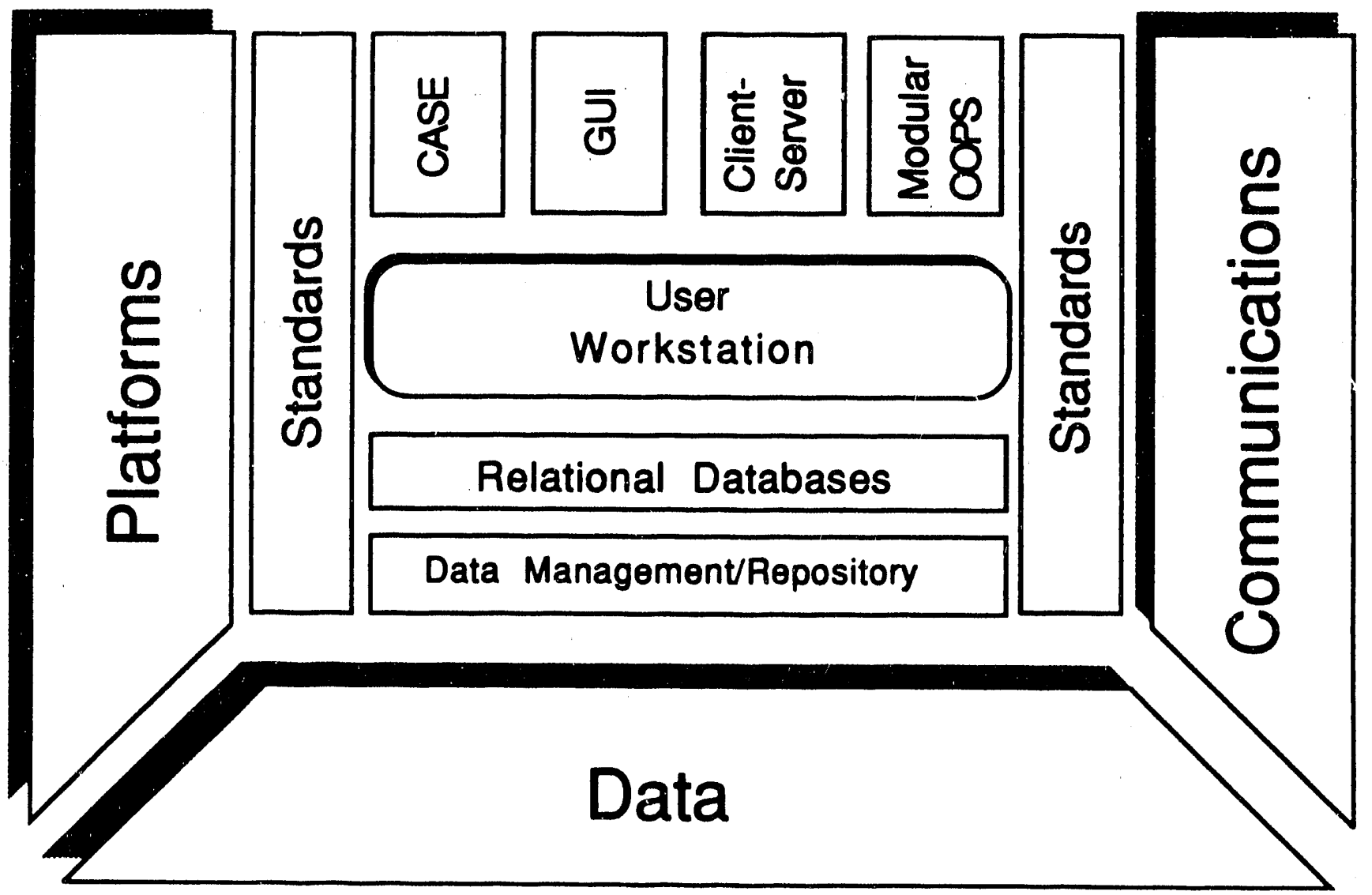

The SRS Computing Architecture

Figure 1 
Implementation of the computing architecture will impact every worker at the site, because it will put in place an environment for accomplishing increasing amounts of routine business functions by electronic means and providing electronic access to large volumes of data. Site management must become more aware of the potential impacts that technical decisions regarding computing systems and applications, data, and communications have on their operations. Adherence to site-wide directions in these areas must be supported if total organizational productivity and quality are to be achieved.

This document is not a call for major increases in funding for computerization initiatives. The site will continue to spend hundreds of millions of dollars on computing each year with or without a common architecture like the one described here. However, adherence to a common architecture is the way to provide common direction for this spending to maximize the benefits of computing as an integral part of fulfilling the site's strategic objectives and mission assignments.

Table 1 summarizes the major points of the SRS computing.

\section{Table 1 \\ Major SRS Computing Architecture Features}

- Standards-based Computing

- Datadriven Systems

- Workstation Orientation

- Automated Applications Development

- Relational Database Technology

- ClientServer Technologies

- Emphasis on Buy vs. Build for Applications

- User-based Data Reporting Tools

- Modularity and Object Oriented Programming

- Site-wide Coordinating Organivzations

This document will re updated annually to reflect changes at SRS and in computing technologies. 


\section{Introduction}

\section{Purpose}

An architecture is proposed here to serve as a unifying plan, or blueprint, for the implementation of computer-based systems and the computing, communications, and data management infrastructure required to support these systems. The value of this architecture (or any architecture) is not so much that it forecasts the emergence and implementation of new computing technologies, but rather that it provides a framework to ensure a consistency in the implementations of individual information systems by the many people across the site invoived in that effort. Only after such a site-wide consistency (or at least harmony) in specific computer technoiogies is obtained can the site integrate the many individual systems into a coherent whole. Achieving this consistency may require that individual implementers of information systems forego the perceived benefits of maximum optimization of computer solutions to individual problems so as to achieve overall computing optimization for SRS.

Fulfilling the site mission in the decade to come will require information resource management to be able to provide site personnel the following capabilities:

- the ability to accomplish most routine business functions electronically

- high availability and reliability of computing services

- a more powerful, intuitive, and universal interface to the end user for accessing computing resources

- ready access to large volumes of information

- the ability to integrate information resources from a variety of sources (including different physical locations, storage formats, organizational etructures)

- more effective communication of information for collaborative efforts

- $\quad$ significantly increased computational capacity

- access to information in meaningful contexts and at multiple layers of detail

- the ability to access and manipulate data in multiple electronic formats including text, graphics, inages, and other data storage forms 


\section{Architecture Goals}

The goals that the computing architecture must support are summarized below. Each component (strategy) of the site architecture satisfies one or more of these goals.

Appendix B relates the strategies of Section 2 to these goals.

I. SRS computing resources must be more effectively utilized.

II. Applications and computer systems must be easier to use.

III. The development of applications must be efficient.

IV. Site applications must integrate into a site-wide information system.

V. Data must be stored in such as fashion as to facilitate sharing.

VI End users must be able to know what data is available and have ready access to it.

VII. Site data must have a high degree of integrity.

VIII. The site computing infrastructure must be robust enough to support the current and future site mission requirements.

\section{Site Computing Architecture Overview}

The site computer arihitecture is a frainework for putting in place informations systems, computer technology, and supporting infrastructure for achieving the goals listed above. This architacture can be summarized as one that is standards-based, datadriven, and workstation-oriented with langer systems being utilized for the delivery of needed information to users in a client-server relationship.. These themes will be stressed repeatedly in this document.

Standards will be based wherever possible on open national and international standards, although standardization on dominant proprietary standards and on specific products will be required to reduce the total number of computer products supported at the site to a more manageable set. In other words, the emphasis on standards will be two-fold: standardization on the interfaces required for information systems integration and standardization on specific products to a more manageable set.

Site data will be uniformly managed. A high-level site-wide organization is required to establish policy concerning the collection, storage, and dissemination of data. The strategic site data must be organized into a model that describes SRS data entities and their relationships. This model will be implemented in a repository, which is a database about databases. 
The user of computer systems utilizing an intelligent workstation, or personal computer, participating in a site-wide computer network will become the central focus of the computing architecture. The user and the workstation become the client, and are supplied information by various information servers. The major difference from today is that the user will see the site information services as a single system via the consistency enforced by standards and the power of the workstation.

Figure 2 below illustrates the central position of the workstation under the client-server paradigm.

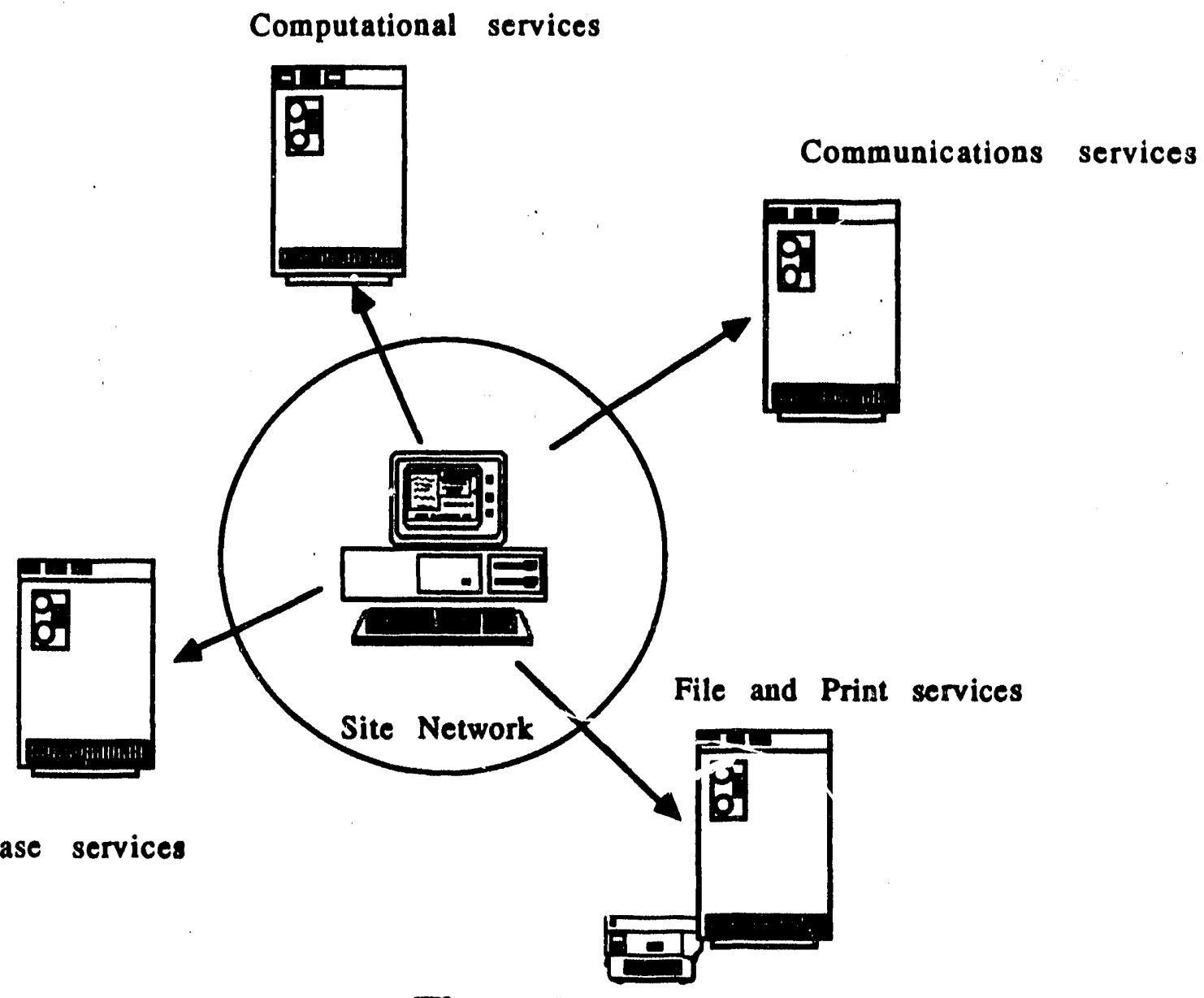

Figure 2

The Workstation is central to client-server computing.

SRS must have one comprehensive computing architecture that is relevant for such diverse areas of computing as systems for management control, design and 
engineering, research and development, operational support, process control, office automation, and management information systems. This is an very ambitious undertaking, as these areas affect virtually all aspects of work at the site. This initial effort at defining the site architecture is focused on areas most applicable to traditional computer information systems. However, the architectural principles and directions put forth in this document are considered applicable to all areas of computing. Future development of the Computer Architecture, under the leadership of the site Computing Architecture and Standards Team (CAST) and involving those most knowledgeable in these areas, will expand the architecture to address any unique requirements of scientific and engineering computing, process computing, and classified computing applications.

\section{Benefits}

Savings and cost avoidance due to the implementation of the computing architecture are potentially in the millions of dollars. (Reference 1 ) These benefits will accrue primarily from the improved productivity of the site's knowledge workers. Site personnel will be given increasingly more powerful and easy to use tools to find and manage the data required for them to accomplish their jobs. The architecture will put in place the environment for accomplishing more and more of the site's business and interactions electronically with the resultant reduction in the "information float" period.

Another significant benefit will be the reduction in duplication of effort in building computer systems and the expense of constructing ad hoc interfaces for computing systems as increasing amounts of the site's computing effort is guided by common principles and standards and an accepted strategic plan for site computing.

New site mission requirements and changing directives and regulations will require improved computing and communications capabilities on the site. That is, new requirements will force new ways of doing business. Increasingly the site faces requirements for:

- more timely reporting of data and reporting of data in ever greater detail

- increasingly sophisticated and complex analyses, technical computations, and process monitoring and control systems

- increased interoperability of previously separate systems

- the ability to transfer graphical and image-based information across the site.

The site will be forced to go to new computing technologies to meet these requirements. The major henefit of this architecture is that it anticipates these requirements and lays out a strategic direction and coordinating mechanisms to get the site in the position to be able to respond to new requirements in a cost effective and efficient manner.

Appendices D, E, and F present various user scenarios that describe how site personnel will benefit from the architecture. Appendix $G$ describes some newer technologies that will evaluated for inclusion into the architecture as they mature. 


\section{Migration Planning}

A migration plan will be developed to guide the transition from the current site environment to that which is specified in this architecture specification. This document will take into account expected availability of technology, existing installed base, and interdependencies among architectural components, and will include cultural and organizational items as well as technological ones. Where this architecture document is generally strategic in nature, the migration plan will be specific and lay out the tactical objectives that must be met to obtain the overall strategy for site computing.

Migration plans will lay out timing of what can be accomplished towards achieving the site architecture based on availability of the technology and interrelationships between components. Individual organizations will be responsible for developing their own implementation plans based on the overall migration plan. These implementation plans will factor in organizational budget, staffing, and workloads in determiring architecture implementation pace.

Migration plan will be published in phases over the course of the next two years, since the implementation path is better defined for some technologies than others.

\section{Management Considerations}

Site management must become more aware of the potential impacts that technicial decisions regarding computing systems and applications, data, and communications have on their operations. Adherence to site-wide directions in these areas must hes supported if total organizational productivity and quality are to be achieved. Tilkewise, the level of redundant effort in the computer area must be reduced. Managers must also ensure that information resources are adequate to support their organizations' responsibilities, and that proper technical decisions are made with regard to information creation, dissemination, and retention.

The architecture and its capabilities will provide many opportunities for improved business and operational methods, but the architecture itself cannot create the change. Initiatives must come from the SRS Computing Council, CAST, and the specific line organizations. Managers and staff must evaluate how they are doing business and make a commitment to continuously improve productivity and quality through application of information management capabilities.

\section{Challenges}

There are several challenges to implementing this architecture. They can be grouped in the areas of cost, redundancy of effort, resistance to change, security, technical management difficulties, immaturity of some of the new technologies and industry standarde, and the overall complexity of the site. These are discussed in the body of this document. 


\section{Strategy Statements}

For the purpose of organization, the statement of the SRS Computing Architecture is organized into several key strategy statements . Strategies are grouped according to the following categories:

Standards

Data

Platforms (systems and workstations)

Communications

Applications

Appendix A lists the strategy statements.

More detailed strategy statements follow that include key components of the strategy, benefits derived from the application of the strategy, and challenges to be faced in implementing the strategy. Additional details are included in associated narrative descriptions. 


\subsection{Strategy Statements for Standards}

The use of standards reduces the total number of variables in the equation for integrating individual systems into an enterprise-wije information system. Furthermore, the use of standards limits the total number of computing products in use at the site and thus concentrates resources on the support of those products which are part of the site standards. Standards are required in all aspects of building a computing infrastructure and the system development process. Requirements for standards include:

- Hardware platforms (Computers and control equipment)

- Operating systems

- Languages (compilers, fourth generation languages, data access, application front-ends, control languages)

- Database management systems

- Data models / data repository

- User interface (includes process operator interfaces)

- Communications networks and system interfaces

- Graphics displays

- Information formats

- System development methodologies

There are two strategy statements that emphasize this direction:

S1 A site-wide standards infrastructure will be established.

$S \quad$ Where applicable, site standards will conform to international and national standards as well as government regulations and DOE orders. 


\section{Strategy}

S1 A site-wide standards infrastructure will be established.

\section{Key Features}

Site-wide Computing Council formed to set policy and address computing priorities.

Computing Architecture and Standards Team (CAST) formed to recommend and implement policies and establish site computing architecture and standards.

Creation of computing committees representing organizations and specialized areas of interest.

Site-wide standards and guidelines developed for computing technologies, methodologies, and products.

Process Digital Equipment Committee to lead establishment of site standards for control and process monitoring systems.

Engineering and Scientific Computing Committee to lead establishment of site standards for engineering and scientific software.

\section{Goals Supported}

I. SRS, computing resources must be more effectively utilized.

IV. Site applications must integrate into a site-wide information system.

\section{Challenges}

Reconciling product standardization with procurement regulations.

Cost of migrating from existing environment to one based on site architecture.

Incorporating systems imposed from the outside.

Encouraging sub-optimization on a local basis in favor of site optimization.

Maintaining an anvironment which encourages creativity. 


\section{Description}

A site-wide Computing Council is being formed to establish site policies relative to the procurement and use of computers at the site and to address the priority of site computing initiatives. This organization will consist of upper management representing each site division. It will oversee the development and implementation of the site computing architecture.

The working arm of the Computing Council will be the Computing Architecture and Standards Team (CAST). CAST is made up of representatives from all site divisions as well as other organizations that represent communities of interest in computing. CAST membership will be knowledgeable in computing technologies and the use of computing to help perform the site's mission. This body will be instrumental in developing standards for site computing, creating the site's computing architecture, as well as recommending computing policies to the Computing Council for consideration.

Currently, there are several division level computing committees and computer user groups which come together to coordinate computing policies and programs at lower levels. These organizations serve to provide valuable input to CAST as well as acting as conduits for relaying proposed cnmputing architecture, policies, and standards to site personnel.

The Process Digital Equipment Committee (PDEC), a long established site body with CAST representation, will lead the development of standards for manufacturing or process computing control systems.

The Engineering and Scientific Computing Committee, also represented on CAST, will lead the establishment of site standards for engineering and scientific software. 


\section{Strategy}

S2 Where applicable, site standards will conform to international and national standards as well as government regulations and DOE orders.

\section{Key Features}

Standards designed to promote interoperability of heterogeneous systems.

Adherence to Government Open Systems Interconnect Profile (GOSIP) as required by DOE order.

ISO and IEEE standards for local area network communications.

Use of dominant proprietary standards and specific products required where open standards are not yet mature.

\section{Goals Supported}

I. SRS computing resources must be more effectively utilized.

IV. C. applications must integrate into a site-wide information system.

\section{Challenges}

Cost and effort to convert existing systems.

Lack of mature product offerings for implementation of standards. 


\section{Description}

Standards will be based wherever possible on open national and international standards, although standardization on dominant proprietary standards and on specific products will be required to reduce the total number of computer preducte supported at the site to a more manageable set. In other words, the emphasis on standards will be two-fold: standardization on the interfaces required for information systems integration and standardization on specific products to a more manageable set.

The primary reason for computing standards is to promote interoperability of the site computing systems. Various international and national bodies are developing so called "open" system standards for computing to promote non-proprietary means of achieving this sort of interoperability. The United States Government, in general, and the DOE in particular are supporting these standards. As these standards mature, the government will mandate their use at government facilities.

The first such requirement from DOE is adherence to the Government Open Systems Interconnection Profile, or GOSIP. GOSIP requirements are based on the work of the International Standards Organization (ISO). Today, GOSIP capabilities are immature, but the final vision of GOSIP is to have non-proprietary standards for many aspects of computing, particularly in the connection of heterogeneous computing systems together into an information network.

The Institute of Electrical and Electronics Engineers (IEEE) is very active in setting national standards for communications (IEEE 802 Local Area Network Standards) and computer operating system interfaces (Portable Operating System Interface or POSLX). Likewise, various vendor consortia, such as the Open Systems Foundation (OSF) are developing and promoting vendor-independent standards for many aspects of computing.

CAST will develop site standards for the computing infrastructure and products that encourages a movement towards more open systems by use of various non-prop ietary computing interface standards. Where there are conflicts in computing interface standards (Often there are two or more proposed standards for each aspect of computing.), CAST will evaluate the competing standards and choose those most appropriate for site use. 


\subsection{Strategy Statements for Data}

Data is the key component of the SRS computing architecture in that data is the most stable component of computing as contrasted with site processes and computing technologies. Data used at the site includes computer databases and files, documents and records, drawings, and images.

Strategy statements in this area are:

D1 Data will be managed as a site resource.

D2 A relational database product will be implemented on the mainframe tier and the workgroup tier. Connectivity will be established between the products.

D3 A site-wide data repository will be implemented for the storage of information describing site data and databases.

D4 Referential integrity will be imposed by the RDBMS wherever possible, instead of by individual applications.

D5 A site-wide data administration organization will be established.

These are described in greater detail on the following pages. 


\section{Strategy}

D1 Data will be managed as a site resource.

\section{Key Features}

Identification of mission-essential data.

Establishing robust platforms, including workgroup servers as well as central computer systems, for mission-essential data.

Uniform standards for data collection, storage, and interchange.

Maximum sharing of data, with appropriate protection constraints.

\section{Goals Supported}

I. SRS computing resources must be more effectively utilized.

VI. End users must be able to know what data is available and have ready access to it. VII. Site data must have a high degree of integrity.

\section{Challenges}

Overconing cultural bias toward proprietary data.

Massive amounts of data being generated at the site.

Constantly changing requirements for data.

Great effort required to manage data in a heavily distributed environment. 


\subsection{Strategy Statements for Data}

Data is the key component of the SRS computing architecture in that data is the most stable component of computing as contrasted with site processes and computing technologies. Data used at the site includes computer databases and files, documents and records, drawings, and images.

Strategy statements in this area are:

D1 Data will be managed as a site resource.

D2 A relational database product will be implemented on the mainframe tier and the workgroup tier. Connectivity will be established between the products.

D3 A site-wide data repository will be implemented for the storage of information describing site data and databases.

D4 Referential integrity will be imposed by the RDBMS wherever possible, instead of by individual applications.

D5 A site-wide data administration organization will be established.

These are described in greater detail on the following pages. 


\section{Strategy}

D1 Data will be managed as a site resource.

\section{Key Features}

Identification of mission-essential data.

Establishing robust platforms, including workgroup servers as well as central computer systems, for mission-essential data.

Uniform standards for data collection, storage, and interchange.

Maximum sharing of data, with appropriate protection constraints.

\section{Goals Supported}

I. SRS computing resources must be more effectively utilized.

VI. End users must be able to know what data is available and have ready access to it.

VII. Site data must have a high degree of integrity.

\section{Challenges}

Overcoming cultural bias toward proprietary data.

Massive amounts of data being generated at the site.

Constantly changing requirements for data.

Great effort required to manage data in a heavily distributed environment. 


\section{Description}

Data will be increasingly viewed as site resource that will be shared as needed. Data viewed as essential to carrying out the site mission will be identified (See Strategy D5.) Distributed access to data will become more common as client-server and peer-to-peer technologies mature. Whenever possible, mission-essential data will be stored on platforms accessible by other users. Shared data will be migrated to either the Central Computer Facility (CCF) or to workgroup servers. These systems will be managed in a production fashion according to site guidelines.

Personal computers (other than those used as workgroup database servers) will be used only for single-user, limited-scope databases. The database packages selected for personal computer databases should provide for connectivity with, and migration to, larger supported environments such as workgroup or CCF databases. (See Strategy D2.) They must be sufficiently easy to use that end-users can create their own applications and databases.

Guidelines for the management of systems supporting shared databases will be developed to ensure these systems and data are managed in a uniform fashion and that they provide, as a minimum:

- adequate capacity for the retention of large quantities of data, where required

- high throughput and rapid response time whether providing data to users or to other sysiems

- $\quad$ high physical data integrity and system availability

- data conformance to site standards

- naming conventions for entities and attributes

- custodian specified for each data element

- data elements defined through textual description

- required update frequencies defined

- enforcement of referential integrity and security 


\section{Strategy}

D2 A relational database product will be implemented on the mainframe tier and the workgroup tier. Connectivity will be established between the products.

\section{Key Features}

Transition of mainframe data and applications to a relational environment.

Standardization on a single workgroup relational database management system (RDBMS) product.

SQL database access language.

Linkage of workgroup and mainframe databases.

Transition (or augmentation) of relational database systems to support object oriented programming.

\section{Goels Supported}

I. SRS computing resources must be more effectively utilized.

II. Applications and computer systems must be easier to use.

IV. Site applications must integrate into a site-wide information system.

V. Data must be stored in such as fashion as to facilitate sharing.

VI. End users must be able to know what data is available and have ready access to it.

VII. Site data must have a high degree of integrity.

\section{Challenges}

Great diversity in database management systems in use on site.

Need for coexistence of existing systems and databases with new RDBMS's during transition.

Cost in converting existing systems to standard RDBMS's.

Greater utilization demands placed on computer systems by relational databases. 


\section{Description}

Depending on its origin and use, mission-essential data will be stored on the Central Computer Facility computers or on computers dedicated to or controlled hy a single workgroup. (A workgroup can be as small as several people and as large as a whole division.) Databases, whether on the CCF or on a workgroup computer, will increasingly use relational database technology.

The strategy for storing data on workgroup computers is to decouple the database management system (DBMS) selection from the hardware and operating system selection by using a single relational DBMS product (RDBMS) which will execute on multiple platform types. This allows the site to take advantage of emerging computer technologies with improved price/performance characteristics while preserving the existing database investment.

The relational database management system for the workgroup tier must also fully support access via the Structured Query Language (SQL) standard, must be suitable for developing software on one hardware / operating system and running it on another, and must provide for transparent data exchange between workstations, servers, and the existing IBM mainframe RDBMS, DB2. Increasingly, applications developed on the IBM mainframe will use DB2 instead of being based on non-relational DBMS.

The site network will connect the standard workgroup RDBMS systems to each other and to DB2. Site standards for open network protocols (TCP/IP and OSI) will provide the transport to link the datahase systems together.

CAST will evaluate various RDBMS and choose via an open, competitive procurement process a product that meets the requirements listed above to be the site standard workgroup RDBMS.

When the future standard, Remote Data Access (RDA), for the interoperability of multiple RDBMS matures, this standard will be adopted at SRS. This will lessen the need for standardization on a single RDBMS.

Object Oriented Databases (OODB) are just now becoming available for use. It is expected that relational database products will be extended in the future to give them capabilities to support Object Oriented Programming Systems (OOPS - See strategy A4). In any event, as object oriented programming moves into the mainstream of site application development, site standards will be required for application development tools and supporting databases that supports the concepts associated with OOPS. 


\section{Strategy}

D3 A site-wide data repository will be implemented for the storage of information describing site data and databases.

\section{Key Features}

Consolidation of meta-data stored in independent data dictionaries.

Accessible by all CASE and DBMS products.

Implements site data model.

\section{Goals Supported}

III. The development of applications must be efficient.

IV. Site applications must integrate into a site-wide system.

V. Data must be stored in such as fashion as to facilitate sharing.

VI. End users must be able to know what data is available and have ready access to it.

\section{Challenges}

Large and diverse amounts of date in use at site.

Lack of availability of single product suitable to implement all repository goals.

Connectivity with related database and applications products. 


\section{Description}

A repository is defined as any database which stores data definitions, code, program design and/or specification objects used in the analysis, design, and generation of application systems.

A data repository containing data describing site mission-essential data will be developed so that information workers can easily lncate desired data and multiple applications and databases can be interrelated without redundant or contradictory data. Mission-essential data will be modeled, with entity, attribute, and relationship meta data (data which describes data) being loaded into the repository.

Automated application design and development tools will be integrated with this data repository, which will facilitate rapid systems application development. Mechanisms for populating data dictionaries used by other database products from the central data repository will be developed as required. 


\section{Strategy}

D4 Referential integrity will be imposed by the RDBMS wherever possible, instead of by individual applications.

\section{Key Features}

Provides consistent implementation of integrity rules across applications.

Derived from rules stored in repository.

\section{Goals Supported}

VII. Site data must have a high degree of integrity.

\section{Challenges}

Implementation in a distributed database environment.

Requires mature repository implementation for consistency.

Inconsistent levels of integrity for existing data. 


\section{Description}

All data has business rules associated with it. These rules describe the range of values acceptable for the various data entities and the relationships of the data entities to each other. Development and documentation of these rules is part of the process of modelling data. A site data repository is the ideal place to store these rules.

Maintaining data integrity (All stored data is correct, and all necessary data is stored.) is essential for any database. This integrity may be enforced by rules coded in individual applications (programs) or by the capabilities of the database management system itself. If rules are enforced in each application, there is no guarantee that they are implemented correctly, or at all, since different organizations accessing data may not be aware of, or care about, all of the integrity rules.

If rules are enforced by the database, development effort is reduced since application programmers don't have to define and code all the rules. Maintenance effort is also reduced since there is only one copy in one location to be modified. Database-enforced rules are better suited to the client-server model since the rules reside only in the distributed database servers or in the central repository. Central storage also permits better security enforcement. 


\section{Strategy}

D5 A site-wide data administration organization will be established.

\section{Key Features}

Data Administration Steering Committee with division-level representation to provide senior management oversight of data policies.

Working-level Data Administration Coordination Group to coordinate implemientation of data policies.

\section{Goals Supported}

I. SRS computing resources must be more effectively utilized.

V. Data must be stored in such as fashion as to facilitate sharing.

VII. Site data must have a high degree of integrity.

\section{Challenges}

Overcoming cultural bias toward proprietary data.

Massive amounts of data being generated at the site.

Constantly changing requirements for data. 


\section{Description}

A senior management Data Administration Steering Committee with divisionlevel representatives is proposed to provide senior management oversight of the adequacy and consistency of data administration practices throughout SRS. The Steering Committee will be responsible for:

- $\quad$ Setting the data administration policy for SRS.

- Resolving major interdivisional issues associated with data administration practices at SRS.

- Resolving issues of priorities in the dedication of resources to support data administration objectives.

A Data Administration Coordination Group will be responsible for ensuring consistent application of data administration practice within SRS.

Responsibilities of this group include:

- Preparation and maintenance of an SRS Data Administration Plan, as well as the coordination of its implementation.

- Identification of data which should be controlled as corporate data.

- Objective organization of data from a site-optimal vs. division-optimal perspective.

- Preparation of data standards, naming conventions, protection guidelines.

- Preparation of company-level policies and procedures for data administration.

- Maintenance of site-wide data models, repository, and dictionaries.

- Review of logical database designs, including addition, deletion, or modification of entities, attributes, and/or relationships.

- Assignment of custodial responsibilities for data elements to appropriate organizations.

- Review of tools and methodologies to be used in data administration.

- Consultation on the data administration discipline and assistance in data administration-related activities as assigned or requested.

- Initiating audits of systems for adherence to data administration guidelines. 
Divisional Data Teams, composed of persons knowledgeable about the business areas specific to a division, will be responsible for interfacing between those business functions and the Data Administration Coordination Group. This responsibility includes:

- Specification of data requirements necessary to support the business, including data elements, relationships, retention, validation, etc.

- Assessment of custodian efficacy (for example, data integrity and timeliness)

- Determination that the value of data exceeds the cost of collecting and maintaining the data. 


\subsection{Strategy Statements for Platforms}

There are thousands of computer systems in use at the site. These range in size from the ubiquitous personal computer found on the desk top of nearly every SRS "information worker" (that is, managers, professionals, and clerical staff) to the site mainframe computers, which are dedicated to providing the major computational and data processing resources for the site. The explosive growth in the number and use of systems at the site has paralleled the computer industry growth of the last decade. These systems have been used on the site to provide significant capabilities in controlling our reactors and processes, providing theoretical and engineering calculations, running the business functions of SRS, and managing tremendous amounts of information.

Strategy statements in this area are:

P1 System acquisitions will be based on site standards and guidelines.

P2 Systems and workstations will be closely integrated into the site computer network with supporting services.

P3 Robust mission-essential computing and process control facilities will be maintained.

P4 There will be a hierarchy of shared data storage capabilities provided for the site.

These are described in more detail on the following pages. 


\section{Strategy}

P1 System acquisitions will be based on site standards and guidelines.

\section{Key Features}

Open systems encouraged by site standards.

Increasing use of the UNIX operating system.

Acceptance of multiple standards for personal workstations.

Identification and implementation of standard mechanisms for linking heterogeneous systems together at the application and database levels.

Site standards for system services that promote interoperability.

Site standards for process control and monitoring systems.

\section{Goals Supported}

I. SRS computing resources must be more effectively utilized.

III. The development of applications must be efficient.

IV. Site applications must integrate into a site-wide information system.

\section{Challenges}

Large numbers of older, proprietary computer systems in use at the site.

Competition by vendors and standards organizations to set standards.

Continuing requirements for the use of proprietary systems based on external or internal programmatic needs. 


\section{Description}

The procurement of computer systems and workstations will conform to standards established under the CAST. PDEC will be responsible for establishing standards for process control and monitoring systems. ESCC will lead in the scientific/engineering area. Each organization will promote standards that provide for "open systems", that is systems that provide for data sharing and application interoperability in a heterogeneous environment and which provide for increasing portability of applications across multiple platforms. Deviations from site standards, where there are compelling justifications, will be allowed, but the planning for the implementation of non-standard solutions must include proposals for the integration of the proposed system into the site information network.

Open systems, in a broad interpretation, are those that conform to international, national, and industry standards. A vendor consortium, supported by the site's major computer vendors, the Open Systems Foundation (OSF) is setting standards for intervendor operability. OSF"s focus is on standardizing UNIX, but the impact of its work goes far beyond UNIX. Today, there are standards for data communications protocols (See the section of this document on Data Communication), user interfaces (e.g. OSF's Motif), operating system services (POSLX), compilers, database access languages (SQL), distributed computing interfaces (OSF's DCE), and compound documents formats (ODIF). There will always be competitive cost and performance issues which separate vendors. But if the majority of our systems conform to a wide range of open standards, the problems of interfacing systems and migration of applications between platforms will be minimized.

Future site standards for computer systems and personal workstations will be developed with consideration given to the large existing installed base. Key computers and operating systems in the current environment that must be supported by the site architecture are:

\section{Computer}

IBM mainframe

DEC VAX minicomputer

Cray

IBM Personal System /2 (and compatibles) Apple Macintosh

Scientific/Engineering workstations

\section{Operating Systems}

\author{
MVS \\ VMS, ULTRIX (UNIX) \\ UNIX \\ MS-DOS, OS/2, UNIX \\ Mac OS, AUX (UNIX) \\ UNIX
}

As noted above, the UNIX operating system is becoming a strategic open system, implemented on computers of all types and with increasing commitment by the vendors to the standards listed above. However, operating systems other than UNIX will continue on the site with increasing openness as they conform to the open system standards. Site standards will increasingly favor open standards over vendor-specific ones. Standards for personal computer software will favor packages that exist for both the IBM Personal System /2 family and the Apple Macintosh systems. 


\section{Strategy}

P2 Systems and workstations will be closely integrated into the site computer network with supporting services.

\section{Key Features}

High performance LAN interfaces for personal workstations.

Establishment of file, printer, and database servers at the workgroup level.

Site standards for workstation communication capabilities and supporting services.

Transition to workstation-based office information systems.

Increasing use of computers and data communications facilities in the conduct of routine site business functions.

\section{Goals Supported}

I. SRS computing resources must be more effectively utilized.

II. Applications and computer systems must be easier to use.

III. The development of applications must be efficient.

IV. Site applications must integrate into a site-wide system.

\section{Challenges}

Great numbers and diversity of systems in use at site.

Costs of upgrading workstations and supporting infrastructure.

Cultural orientation to paper-based systems. 


\section{Description}

The days of "stand-alone" (unclassified) computer systems are nearing an end. Each system, from the smallest desk top computer to the site mainframe computers, will attach to the site network. These attachments will be via relatively high speed local area network (LAN) links. Advantages of this architecture are:

- Required for client-server applications and image-based applications.

- High speed data transfers between workstations and host computers.

- Promotes "groupware", software that empowers workgroups or project teams with tools for work sharing information exchange and office information systems.

- Promotes standards on the desk top systems.

- Gives access to workstations to "Archive Servers" for data backups.

PC LAN standards that support both IBM and Apple systems will be implemented for greater resource and information sharing between the users of these machines.

A major component of the architecture is the concept of client-server interactions for the distribution and presentation of information. Clients are agents that perform tasks on behalf of the user, mediating between the user and the hardware/software system. Servers are objects called upon by clients to do one or more specialized functions to complete a task. Functions can be as varied as performing a complex computation, returning results of a database search, or providing file and print facilities for the client.

Client-server applications provide, above all, a consistency in application interfaces for the user of a workstation resulting in overall ease of use and minimizing training and support requirements. The implications of a client-server architecture for platforms are the following:

- Workstations must attach directly to the LAN.

- Workstations must be relatively powerful. Older personal computers (e.g. IBM PC/XT and PC/AT, Macintosh Plus) must be replaced by more powerful systems.

- "Host" computers will evolve into information and resource servers in addition to their traditional roles as platforms for transaction processing, business reporting, and scientific and engineering calculations.

- Consistency across the site will be provided by standards for graphical user interfaces on the workstations and data access and communications protocols on the workstations and the servers.

- Security procedures on the servers must evolve from today's traditional interactive login security to network-wide authentication via standard security protocols. 


\section{Strategy}

P3 Robust mission-essential computing and process control facilities will be maintained.

\section{Key Features}

Resource utilization forecasting to schedule new systems and equipment upgrades to be available when required to support the site mission.

Disaster recovery plans for central computer facility and other systems essential for site operations.

Establishment of policies to keep hardware and system software on designated systems at levels supported by the vendor.

Process control and monitoring systems to meet prudent as well as required levels of redundancy and fail-safe operation.

\section{Goals Supported}

V. Data must be stored in such as fashion as to facilitate sharing.

VIII. The site computing infrastructure must be robust enough to support the current and future site mission requirements.

\section{Challenges}

Difficulties in accurately forecasting computing needs in a time frame consistent with major ADP equipment procurements.

Disaster recovery plans will require some duplication of equipment, facilities, and services, which must be funded. 


\section{Description}

As site requirements for computing (transactions and reports in management information systems, data storage, scientific and engineering calculations, and process monitoring and control) continue to grow, it is critical that the required computing resources be developed and maintained. Large computers require relatively long lead times to procure. This makes correct and timely identification of the needs for computer resources to support the site mission especially important.

Certain computing functions are so critical to the operations of the site that their loss due to some disaster must be considered in site planning and recovery options planned in advance. In some cases the functions are so critical that separate, duplicate facilities will be required to continue operations in the event of a disaster that takes down the primary facility. In other cases, standby facilities can be established at other DOE sites or commercial facilities that specialize in providing disaster recovery alternatives.

Various regulations, orders, and rules require disaster recovery and planning and define levels of redundancy required for critical systems. It is important that this planning be incorporated into the budgetary process to insure that adequate funding is established in support of the disaster planning.

One danger in a period of budget austerity is that our computer systems become so obsolete as to no longer be supported by the equipment or software manufacturer. Without this support maintenance and modifications of the systems becomes difficult and expensive. 


\section{Strategy}

P4 There will be a hierarchy of shared data storage capabilities provided for the site.

\section{Key Features}

Personal, workgroup, and central data stores.

Shared facilities for cost efficiencies.

Sufficient facilities to provide data backup for all platforms.

Data migration from fastost (most expensive) storage to slowest (least expensive).

Ready access from site standard systems to shared storage.

\section{Goals Supported}

V. Data must be stored in such as fashion as to facilitate sharing.

ViII. The site computing infrastructure must be robust enough to support the current and future site mission requirements.

\section{Challenges}

Reconciliation of current UCNI data access requirements with shared computer storage.

Obtaining adequate funding for shared storage of all the data the site may be required to archive. 


\section{Description}

The Central Computer Facility (CCF) and other large computer systems on site will evolve into information and resource servers for client computers, predominantly personal computers, but also engineering workstations and other large systems. One of the major services that the CCF is uniquely able to provide is that of site-wide "storage server". Regulatory requirements as well as DOE orders and site QA rules are requiring increasing amounts of long term data storage. Much of this data will be centralized in the CCF for the benefits of economies of scale. The CCF will provide a variety of data storage capabilities. For data requiring fast access (i.e. database applications) fast disk drives will be increasingly supplemented by expanded memory storage and solid state "disk drives". For data that must be on line but is infrequently accessed, read-write optical disks with large "jukebox" arrays are possible. For archived data, read-only optical disk can be used. Finally, long term archived data will continue to go to off line storage with digital tape providing increasing storage capabilities over traditional computer magnetic tape.

System and network procedures will be developed to facilitate the movement of data from the individual workstations to the central archives. This will provide automated, unattended backup of each user's workstation to guard against data loss. Development work in the area of providing data retrieval capabilities is required so that data that is archived can be readily found and accessed when required.

Other systems across the site will provide similar storage capabilities for storing data at the workgroup level. 


\subsection{Strategy Statements for Communications}

Data communications (computer networks) provide the capability to link data, applications, workstations, and systems into a comprehensive site information system. This has been an area of explosive growth over the last decade, both in site implementations and technology advances. By the end of 1990 the site computer network consisted of over $\mathbf{4 0 0}$ host computer systems and over 10,000 workstations.

The following are the strategy statements for Data Communications:

C1 Data communications services and products will be based on standards.

C2 The site network will be composed of robust LAN trunks and inter-area (facility) links.

C3 Workstations and host computers will connect directly to the site LAN.

Descriptions of these strategies follow. 


\section{Strategy}

C1 Data communications services and products will be based on standards.

\section{Key Features}

Phase out of proprietary communications protocols in favor of open protocols (TCP/IP and OSI)

Development of a network operations and management infrastructure to support open protocols.

Rework of SRS network backbone to establish separate area networks with communications based on high performance routing of data between areas.

Establishment of site standards for communications products for personal workstations, control systems, and host computer systems.

Development of network-based security features based on industry standards.

\section{Goals Supported}

I. SRS computing resources must be more effectively utilized.

IV. Site applications must integrate into a site-wide information system.

VIII. The site computing infrastructure must be robust enough to support the current and future site mission requirements.

\section{Challenges}

Cost to convert from large number of proprietary communications protocols and products in use at the site.

Management of communications protocols in thousands of individual workstations

Effective use of mandated communications standards which are technically immature. 


\section{Description}

The site computer architecture depends on the establishment and enforcement of standards as a way of simplifying the site computer environment so that the integration of the various components into a cohesive whole is even possible. Data communications protocols have been a subject for national and international standardization for some years now so that site standards can be based on standards declared by government bodies and supported by the vendors. As the technology changes, new standards will emerge so that it is important that a site-wide standards setting body be created and remain active. Current and near term future standards include:

\section{TCP/IP}

In the short term (1-5 years) and before Open Systems Interconnection (OSI) protocol implementations are mature, the dominant protocol for interconnection of heterogeneous systems will be TCP/IP (Transmission Control Protocol / Internetwork Protocol). This set of protocols, created for a major Department of Defense and research community wide area network, called the Research Internetwork, is currently well supported on all major workstation and host computer environments. Importantly, many distributed database applications will be built upon TCP/IP protocols. Computer vendors are developing products that will allow TCP/IP to coexist with OSI and facilitate the eventual replacement of TCP/IP with OSI protocols.

\section{OSI (GOSIP)}

GOSIP stands for the Government Open Systems Interconnection Profile. It is the federal government's requirement for specifying Open Systems Interconnection (OSI) communications protocols for computer procurements. OSI protocols are emerging as a vendor-independent set of computer communications protocols. Products to meet the OSI standard are just now emerging. GOSIP represents the government's view of which protocols within OSI are mature enough to be required in computer procurements. Each year the number and level of OSI protocols and applications will increase. Government procurements of computer systems with file transfer or electronic messaging capability must specify the appropriate GOSIP protocols be made available .

Over the next 5-10 years OSI protocols will become the dominant communications protocol suite on the site network. All our major computer vendors are committed to support them; those that don't will be unable to win procurements at the site.

\section{Propecietary Protocols}

The many proprietary protocols in use at the site today will be gradually phased out in favor of open protocols. Major proprietary protocols in use at the site today include SNA (IBM mainframes), DECnet (DEC minicomputers and workstations), AppleTalk (Apple Macintosh systems), and IPX (Novell Netware personal computer LAN's). 
The diagram below shows the emergence of OSI as the dominant site protocol over the next decade as DECnet and TCP/IP are replaced by OSI and OSI/SNA coexistence is provided by IBM. Note that the protocol set to be supported in the future will be much smaller than today.

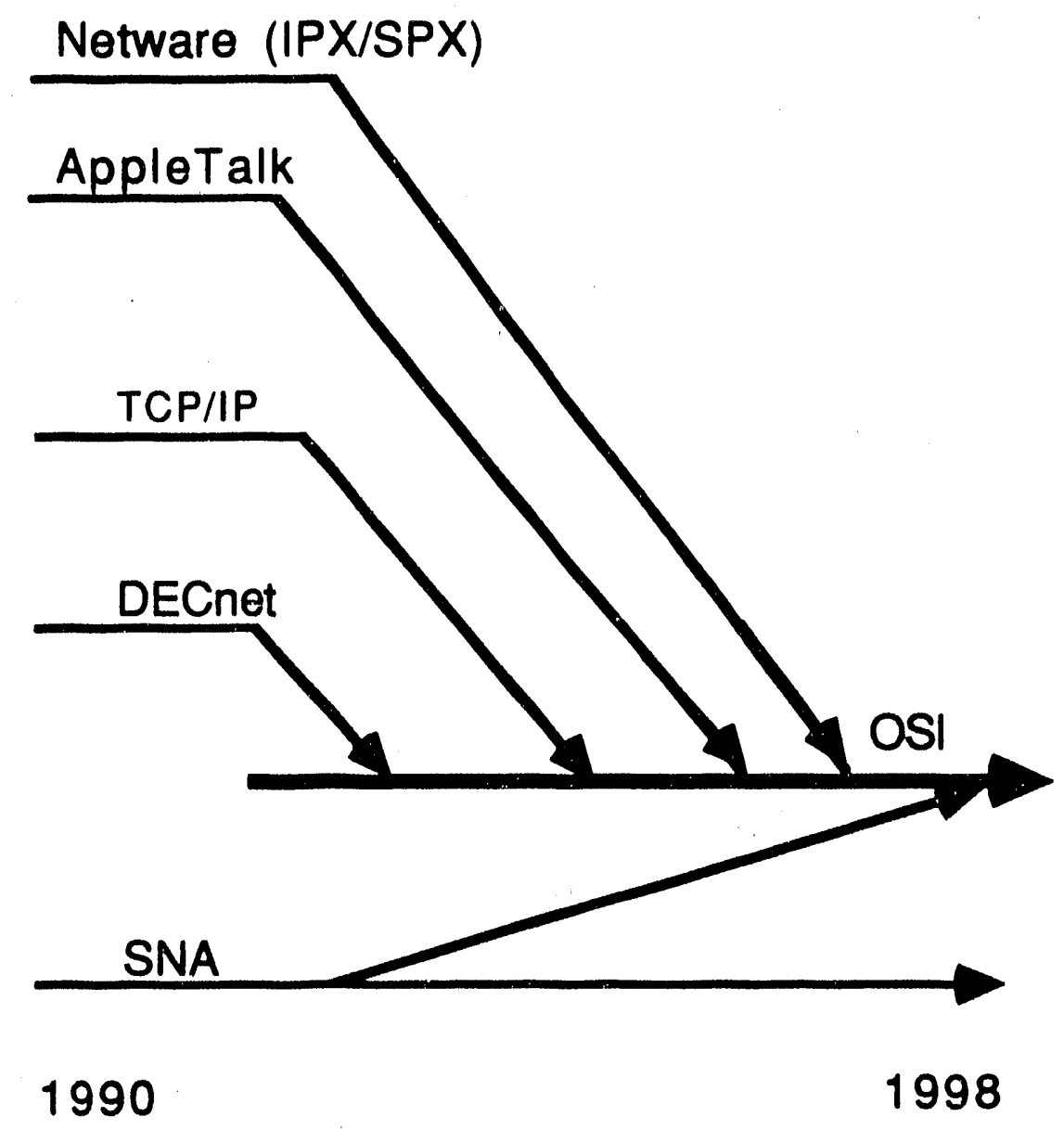

Figure 3

Proprietary Protocols Convergence on OSI Protocols

OSI protocols and GOSIP requirements are discussed in detail in Reference 2.

Site LAN's will conform GOSIP requirements at the physical and link levels of the OSI model. Today most site LAN's are Ethernet (IEEE 802.3). Token Ring LAN's (IEEE 802.5) will be supported for interoperability with the IBM CCF.

In the process area, the Manufacturing Automation Protocol (MAP), or IEEE 802.4, will be investigated for suitability as a site standard. In the future Fiber Distributed Data Interface (FDDI) LAN's will be used to improve the basic throughput capabilities of the site network. Each of these four LAN types is compliant with the GOSIP standard. 


\section{S'rategy}

C2 The site network will be composed of robust LAN trunks and inter-area (facility) links.

\section{Key Features}

Heavy reliance on fiber optic technology for data communication, including

Fiber Distributed Data Interface (FDDI) local area networks and interarea fiber links.

Dedicated facilities for process control LAN's.

Proactive network monitoring and management.

High-speed connections to WSRC/BSRI off-site locations.

Enhanced disaster recovery capabilities.

\section{Goals Supported}

I. SRS computing resources must be more effectively utilized.

IV. Site applications must integrate into a site-wide information system.

VIII. The site computing infrastructure must be robust enough to support the current and future site mission requirements.

\section{Challenges}

High capital cost for major network upgrades.

Complexity introduced by dispersed geographical nature of SRS facilities.

Lack of comprehensive and effective network management tools.

Demands for data by applications growing faster than capabilities of communications technologies. 


\section{Description}

As site information applications become increasingly dependent on the site LAN, it will be upgraded to provide the necessary throughput and reliability. This upgrade will consist of Fiber Distributed Data Interface (FDDI) LAN's in major site areas and high speed interarea links based on fiber optic technology. FDDI supports a basic communications rate of 100 million bits per second (mbps). Interarea links will be at least 45 mbps. Links to major off-site WSRC/BSRI facilities will be at least $1.5 \mathrm{mbps}$.

Existing LAN's will become tributaries to the FDDI LAN's as shown in Figure 4. Upgrades of the existing LAN's will occur over time as the cost of the new technology reduces and as the communications requirements of the individual workstations grow. In the 5-10 year range new technology will emerge to supplant FDDI for the LAN trunks. This technology will be fiber optic based and will support at least one gigabit data transmission rates.

Because of the complexity of the current and future site networks an aggressive and proactive network management program is required, Such a program will require documentation of all network interfaces (down to individual workstations), proactive network monitoring, and network audits. Network management tools will emphasize standards, Simple Network Management Protocol (SNMP) for TCP/IP network entities and Common Management Information Protocol (CMIP) as part of the transition to GOSIP.

Many of the proprietary process control "data highways" are being converted to standard LAN technology such as Ethernet (IEEE 802.3) or MAP (IEEE 802.4). Such LAN's will remain dedicated to the process function and will not share facilities with the common area LAN. However, it is desirable (where security permits) that controlled gateways link the process LAN's and components to the general site network. Such gateways must allow the flow of process data to reporting databases, but limit traffic from the site LAN to the process LAN to protect the integrity of the process. 


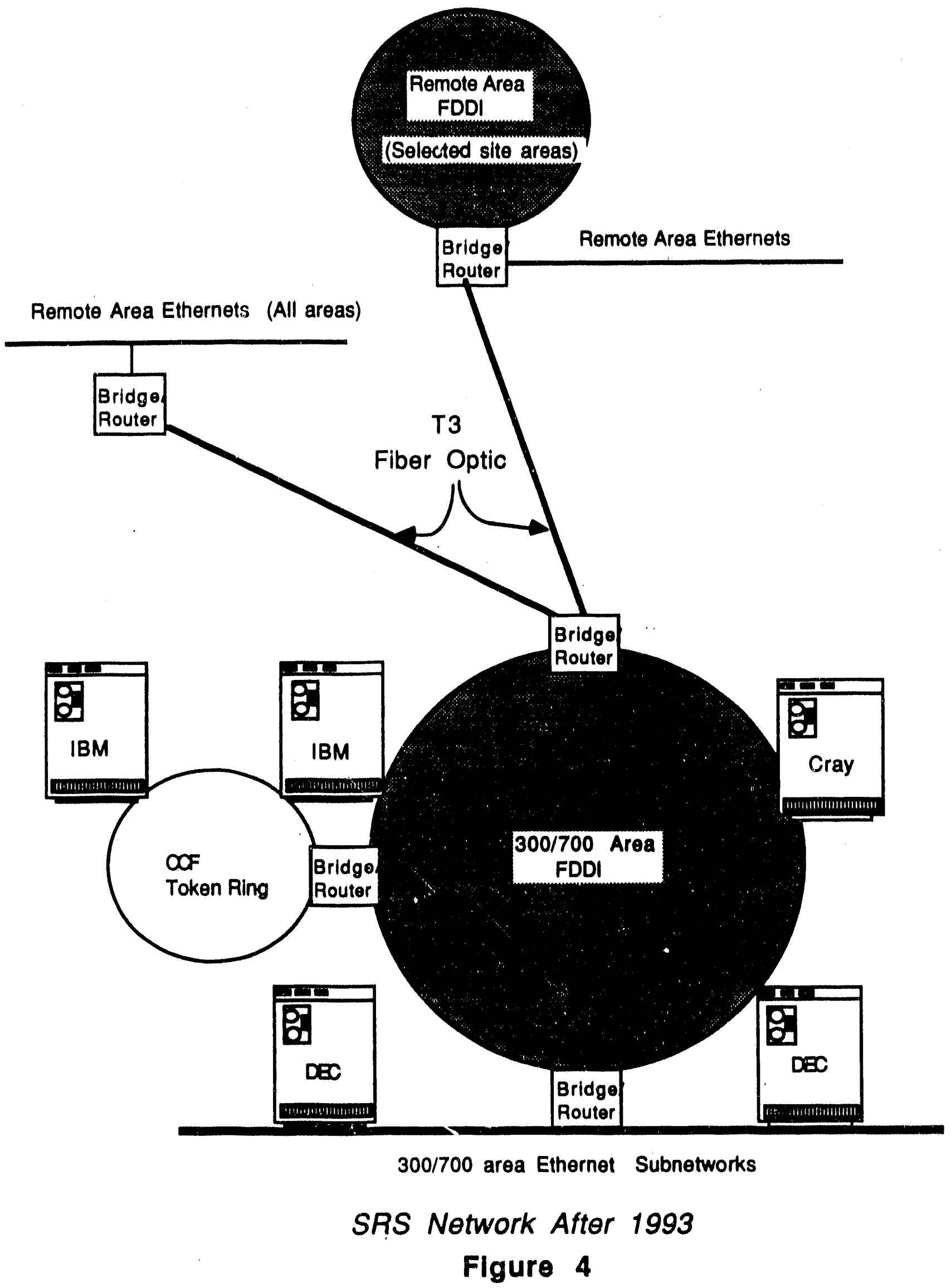




\section{Strategy}

C3 Workstations and host computers will connect directly to the site LAN.

\section{Key Features}

Migration of workstations from serial to direct local area network connections.

Convergence of central computer facility network with site local area network.

Standards in communications protocols to permit maximum interoperability between diverse workstations and systems.

Phase out non-intelligent $v$ orkstations (terminals).

\section{Goals Supported}

I. SRS computing resources must be more effectively utilized.

II. Applications and computer systems must be easier to use.

III. The development of applications must be efficient.

IV. Site applications must integrate into a site-wide information system.

VIII. The site computing infrastructure must be robust enough to support the current and future site mission requirements.

\section{Challenges}

Great number of workstations with only slow-speed terminal access to site networks.

Current dual nature of Central Computer Facility network and site LAN.

Upgrades of older building wiring required to permit direct LAN access. 


\section{Description}

Most workstations today are connected a low speeds ( $~ 9600$ bits per second) to terminal servers or to the CCF terminal control units. New applications will demand increasing bandwidth to the workstation. This bandwidth will be supplied by linking each workstation directly to the site LAN. Site host computers will also connect directly to the LAN, and adherence to communications standards will insure that each workstation will be able to communicate with any required host-based application or database. Speeds between the LAN and the personal computers will be at least $1 \mathrm{mbps}$ and will approach 10-50 mbps in specialized applications such as full motion graphical displays of scientific or engineering calculations.

Each personal computer will be assigned a "home" file server so that application and communications software can be centralized in the server for access by the client workstations. This will require a degree of standardization in software and configurations on individual personal computers, but will greatly simplify support requirements for personal computer users. For example, when a standard personal computer software package upgrades, the copy of the new version can be placed on the file servers for each workstation to access rather than requiring each user obtain the upgrade independently. This standardization is also required to insure that applications based on communications between site computers and the personal computers function reliably.

Each "home server" will be located geographically close to the user community it supports. This will limit the amount of data that must cross network segment boundaries and thus stress network trunk facilities. In cases where workgrouris are Geographically dispersed, the users will also have access to a common server( $(s)$ for sharing of information.

Figure 5 shows the personal computers on the LAN with access to host computer services. Host services will include interactive terminal access to host-based applications, file transfers for movement of information between systems, distributed database applications, distributed computational applications, and communications services for both within and outside the site. PC-based file servers will be established that can support both IBM and Apple personal computers and will provide data sharing among these systems in addition to being the vehicle for keeping individual systems standard. 
Information May Be Distributed Within The System at the Personal, Workgroup, and Corporate Levels

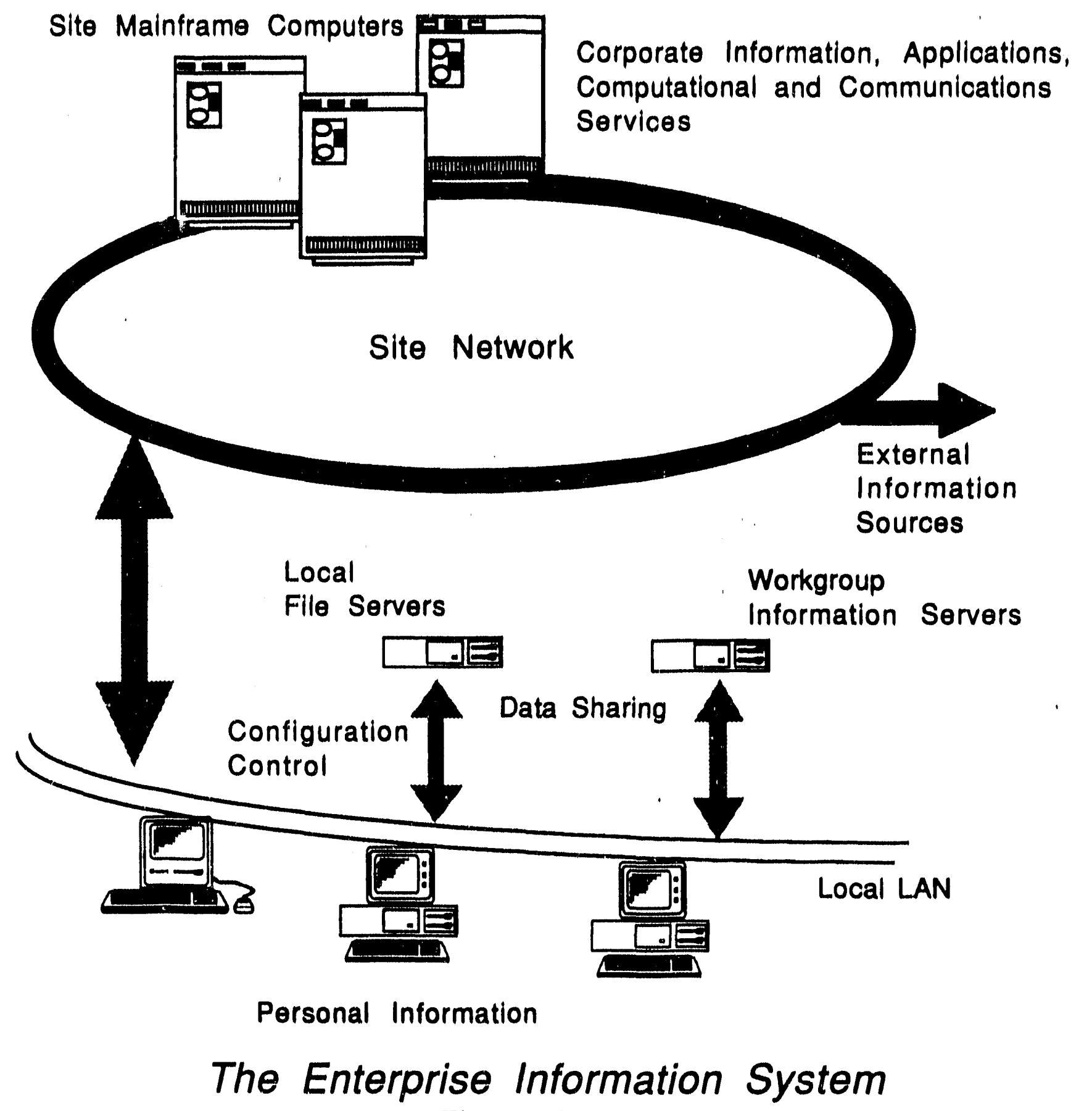

Figure 5 


\subsection{Strategy Statements for Applications}

The term applications in the context of information systems technology refers to the computer programs (software) which provide for the creation, processing, storage, and retrieval of information (data). Applications may be written by site personnel, or they may be purchased from outside vendors. Applications may be specific to the site, or they may provide a generic business or engineering function. Applications may be small, such as software which supports a single individual, or as large as a sitewide information system. Applications require computer hardware to run on (the platform) and a operating system for the hardware. Many applications rest on a base of support software such as a general purpose database management system, communications software, and software which controls the inputs to and outputs from the computer. These supporting technologies are discussed earlier in this document.

Under the site computing architecture, the distinction between Office Automation (OA) and traditional information systems (IS) or data processing (DP) disappears.

Traditional OA systems, like future IS applications, promote the movement of information across an enterprise in electronic format. Even today, relatively few systems are dedicated to traditional OA tasks such as word processing. Instead, general purpose systems are used for these tasks. Technology trends in information systems described below such as client/server relationships apply equally well to OA functions.

Application strategy statements are:

A1 The development and purchase of software will be based on site standards.

A2 Off-the-shelf software will be used to the maximum extent possible.

A3 CASE and other computer-based tools will be used to automate the building of applications.

A4 Applications will become modular.

A5 Applications will conform to site standards for common graphical user interfaces.

A6 The capabilities of the intelligent workstation will be a key component of the architecture.

A7 Users will be provided with tools to enhance their ability to generate their own database reports and queries.

These are described in greater detail below. 


\section{Strategy}

A1 The development and purchase of software will be based on site standards.

\section{Key Features}

Standards included in site software acquisition and development procedures.

Site standards for languages and development tools.

Standards to promote cross system interoperability.

Development of an application migration / conversion policy.

\section{Goals Supported}

I. SRS computing resources must be more effectively utilized.

II. Applications and computer systems must be easier to use.

IV. Site applications must integrate into a site-wide system.

\section{Challenges}

Great number and diversity of development languages and tools in use at site.

Large number, diversity, and complex relationships of existing site applications.

Nonstandard applications and systems imposed on the site from outside.

Acceptance of site standards by application developers. 


\section{Description}

The use of standards reduces the total number of variables in the equation for integrating individual information systems components into an enterprise-wide information system. The use of standards also limits the total number of computing products in use in the application development process and thus concentrates resources on the support of those products which are part of the site standards.

Standards are required in all aspects of the application development (or purchase of application software) process. Many of these have been discussed elsewhere in this document, but it is helpful to list them again here. Standards are required for:

- Hardware platforms

- Operating system (and system calls available to developers)

- Languages (compilers, fourth generation languages, data access, application front ends)

- Database management systems

- Data models / data repository

- User interface

- Network interface (user access, calling of remote procedures, remote data aciess)

- Graphics displays

- Information formats

- System development methodologies

The site Computing Council through its working arm, the Computer Architecture and Standards Team (CAST), will set standards in these areas. Because of the great rate of technological change in the computing industry today, this group will have to constantly monitor the state-of-the-art in information systems and the computing requirements of the site. The establishment of standards will always be a series of trade-offs; any attempt to limit the number of products used on the site for overall simplicity and ease of integration means that individual problems may be solved in less than optimum (considering any single problem in isolation) ways. The use of standards also requires a cultural adjustment by site computing personnel, as each organization gives up individual autonomy and freedom to choose particular solutions to their problems. 


\section{Strategy}

A2 Off-the-shelf software will be used to the maximum extent possible.

\section{Key Features}

Incorporate make versus buy decision into site standard Systems Development Methodologies.

Take advantage of industry-standard applications / practices.

Deliver greater functionality for a given amount of effort.

\section{Goals Supported}

I. SRS computing resources must be more effectively utilized.

III. The development of applications must be efficient.

\section{Challenges}

Many site processes and business procedures are viewed as unique.

Incorporation of purchased software often requires adjustments in some aspect of how the site's business is run.

Purchased software often requires major customizations to be accepted at the site. 


\section{Description}

While CASE technology and impmyed development tools can go a long way toward expediting software delivery, the scope of new efforts indicate that the implementation of applications must occur on a much more rapid time frame than even these improved methodologies can support. Additionally, the cost-effectiveness of developing custom solutions to business needs which are in the process of aligning themselves to 'standard' industry practices must be questioned. "Off-the-shelf" application software packages will be used to the maximum extent practical, in deference to the building of custom solutions. Information systems personnel would concentrate on requirements analysis, product selection, and integration of the application into the SRS environment. Implemented correctly, this will result in decreased development times, reduced application backlogs, and decreased long-term support efforts.

For such a program to be successful, however, packages must be evaluated critically to ensure compliance with architectural specifications and compatibility with guidelined products. More importantly, there must be management support to adapt business practices to conform to process models supported by available software packages. 


\section{Strategy}

A3 CASE and other computer-based tools will be used to automate the building of applications.

\section{Key Features}

Automation of Software Development Methodologies.

Active links to site data repository.

Application development centered at the workstation.

Software configuration management.

Data-driven process control systems.

\section{Goals Supported}

III. The development of applications must be efficient.

V. Data must be stored in such a fashion as to facilitate sharing.

\section{Challenges}

Current tools embryonic and not fully integrated.

Training requirements for application analysts and software developers.

No single CASE tools supports all development environments. 


\section{Description}

The benefits of the computer age must be brought to those who develop computer applications. Today, applications are often designed and coded the same way they were before the advent of the personal computer a decade ago. The personal computer can be a tremendous productivity aid for the application developer just as it has proved to be for the engineer and for knowledge workers in general.

Computer Aided Software Engineering (CASE) tools are largely based on the power of the $\mathrm{PC}$ and are used to proceduralize and automate the planning, analysis, and design of computer applications based on structured development techniques. These tools automate the production of data-flow, decomposition, and entity relationship diagrams and verify consistency across diagram types. Future CASE tools (so called Integrated CASE, or I-CASE) take this automation a step farther by interfacing the tool with the enterprise data model as implemented in the site data repository and generating the code from high-level design specifications. I-CASE will also facilitate the reduction of applications to a set of modules (or objects) and the cataloguing of these modules within the site repository for maintenance control and use as building blocks for future applications. One feature of CASE that makes it such a useful productivity aid for system designers and programmers is that CASE concepts and tools encourage prototyping for rapid application development and feedback from the customer on application fidelity.

CASE will also require a large commitment to change by the application developer. The benefits of automation, like factory automation, can only occur if management is committed to it and the workers are effectively trained in its use. Since the software development "factory" will not be shutting down for a conversion, the evolution will be that much more difficult. Today, the current tools are embryonic and will only evolve to full I-CASE potential by the later half of the decade, but the concept is well established and early products are available. Site application designers and code developers must begin indoctrination in the use of CASE today in order to be ready to apply the more robust tools of the future.

Application development is usually a team process. Personal computing and LAN technologies can provide the "groupware" utilities to help a group build an application. These include shared document composition, on line documentation, electronic messaging, code management systems, and a code test environment that is isolated from the production system but duplicates its behavior.

Emphasis will be placed on acquiring control systems that minimize the requirements to create large quantities of application software to meet functional requirements. Such systems will use configuration databases and parameters to customize the control process. 


\section{Strategy}

A4 Applications will become modular.

\section{Key Features}

Applications as collections of functions that can be accessed independently.

Functions, or modules, defined to a site repository to facilitate reuse.

Development of linkage of major application systems via cross-system procedures that can work across the site network (Remote Procedure Calls or RPC).

Object-oriented programming.

\section{Goals Supported}

I. SRS computing resources must be more effectively utilized.

III. The development of applications must be efficient.

IV. Site applications must integrate into a site-wide system.

V. Data must be stored in such as fashion as to facilitate sharing.

VII. Site data must have a high degree of integrity.

\section{Challenges}

Migration of existing monolithic applications to modular form.

RPC technology is still emerging with no single clear standard implementation.

Immaturity of object-oriented systems and databases. 


\section{Description}

Applications serve the knowledge worker. Today's knowledge worker requires information from a wide range of sources (applications). Basically, the function of the knowledge worker is to gather information from a variety of sources, analyze the data, draw conclusions, make recommendations, and distribute the results. This type of worker requires applications that serve this integration function. Large, single purpose applications present additional barriers to the integration process, as the knowledge worker must learn how to enter, navigate, and exit several separate environments. What is required, are applications that can be thought of as many separate functions, each function independent and usable on its own. Knowledge workers will pick and choose those functions necessary to accomplish the job at hand.

Decomposition of application design into separate functions is what application modularity is all about. Given that individual modules are catalogued in an enterprise repository, modularity encourages reusable code. That is, if a module exists to perform a function, and its existence and use are well documented, then future application developers that require the same functionality will be able to reuse the existing module.

Modular code encourages cross-system integration. If the flow of information that a user of an application requires would lead to a function performed by another application, rather than the user having to exit the first application and enter the second, modular code and implemented standards for remote procedure calls would allow the first application to call and present the results of the second application's function. Suppose, for example, a Works Management user identifies a certain piece of equipment and then needs purchasing data about the equipment. The Works Management application could be written to call the Purchasing function that reports the information about the equipment.

Modularity is also a key component of Object Oriented Programming Systems (OOPS). OOPS represents a programming paradigm shift and is a fundamental break with the application development practices of the past. Under OOPS each data structure is bound together with its associated procedures (or processes that effect the data structure) in a entity called an object, which represents something in the real or conceptual world. Under OOPS applications reduce to a series of predefined procedures operating on data. Since the procedures themselves are embedded in the objects, each application treats the objects and their associate procedures as "black boxes". Applications activate procedures associated with objects by sending "messages" to the objects. Different applications will use the same objects so that the objects themselves represent reusable code.

Modular and object-oriented code also lends itself well to automating rules about data objects through the use of expert systems technology. Rules that govern the relationships of data entities can be made part of the objects themselves. When an event is triggered by a message to an object to complete some process associated with the object, the object can trigger other events based on the rules associated with it. These subsequent events can take the form of messages to other objects. 


\section{Strategy}

A5 Applications will conform to site standards for common graphical user interfaces.

\section{Key Features}

Definition of site standards for Graphical User Interfaces (GUI)

Establishment of GUI-based operating systems for site personal computers.

Inter-application consistency and ease of use.

Migration of some existing applications to GUI-based.

Workstation-supplied GUI for client-server applications.

Multi-media (graphics and images) support under GUI.

Site guideline for process operator displays, alerts, alarms, and interactions.

\section{Goals Supported}

I. SRS computing resources must be more effectively utilized.

II. Applications and computer systems must be easier to use.

IV. Site applications must integrate into a site-wide information system.

\section{Challenges}

Immaturity of GUI development tools, especially those allowing cross-system portability of applications.

Lack of trained GUI programmers.

Cost of adding GUI to IBM PC family.

Large inventory of character-based (non-GUI) applications. 


\section{Description}

The graphical user interface (GUI) popularized by the Apple Macintosh is now accepted by all major platform vendors as the preferred way for users to inter'act with computer applications. IBM and Microsoft have specified Presentation Manager (OS/2) and Windows 3 (MS-DOS) as implementations of a "common user access" while the OSF and X/Open consortiums have specified the Motif "look and feel" for Xwindows workstations. All of these interfaces are basically the same. They are based on graphical displays and "mouse" input pointers, with heavy emphasis on pictures and symbols (icons) to suggest actions by the users. Interaction with an application is object oriented; the user selects an object first then selects an action relative to the object. Common actions such as "determine available options", "select an option", "select from a list of items", and "open or close files" are handled the same way from application to application. Because the user interface is defined and consistent from application to application, new applications are much easier to learn than before. Users feel that use of new applications is intuitive simply because the user interface is familiar. An industry study found that "... experienced GUI users accomplished 58 percent more correct work in the same time [ than users with the traditional character based interface]" (Reference 3).

Applications from different host computer environments appear the same to the user, as each conforms to the same GUI. A useer's workstation will provide concurrent "windows" into multiple applications. The applications may be local to the workstation (such as word processing) or they may access data from one or more host computers. The figure below represents this. It is important to note that the common user interface shared by these applications means that the user need not be aware of the systems and network topology providing the application access. Applications driven by remote host computers will seem to the user as if they are local to the workstation. 


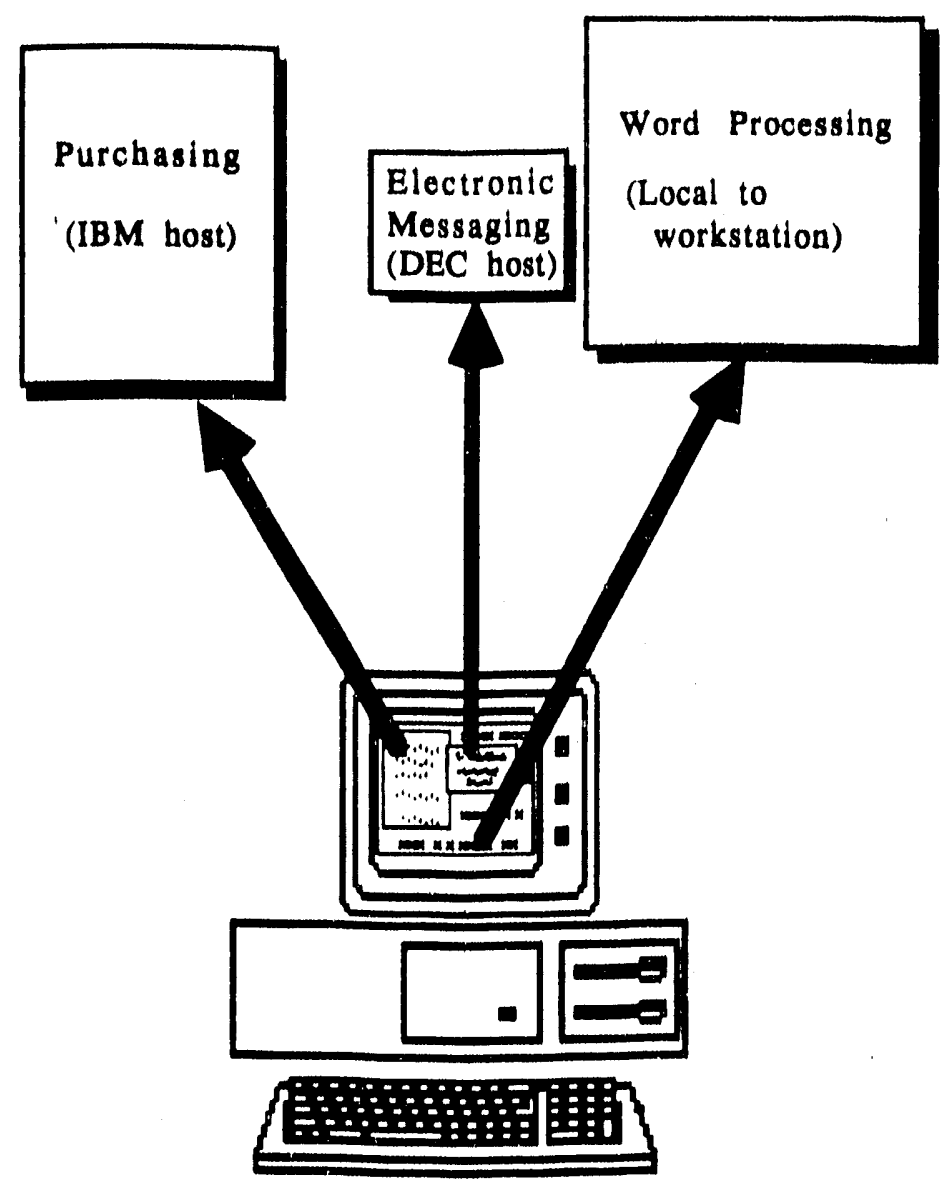

Workstation Access to Multiple Hosts Via Windows

Figure 6

PDEC will develop site guidelines for process operator displays, alerts, alarms, and interactions that will insure that operators have clear and unambiguous picture of the process being monitored, operation attention is focused on those areas that require attention, and operator interaction with the process is as straight forward and easy to do as possible. 


\section{Strategy}

A6 The capabilities of the intelligent workstation will be a key component of the architecture.

\section{Key Features}

Increasing computational power of the workstation to be utilized.

Linkage of workstations directly (high speed) to site network.

The workstation (personal computer) provides client in client-server computing.

Client-server computing to include GUI, remote database access, and partitioning of application via RPC technology.

Workstation-based Office Information Systems (OIS).

\section{Goals Supported}

I. SRS computing resources must be more effectively utilized.

II. Applications and computer systems must be easier to use.

IV. Site applications must integrate into a site-wide system.

\section{Challenges}

Upgrade costs for obsolete workstations.

Complexity of applications spread across multiple platforms.

Immature state of distributed applications tools (RPC and RDA).

Providing a network that can support large increases in data communications requirements. 


\section{Description}

With the intelligent workstation (personal computers and high performance engineering workstations) supplying the interface between the user and the application, the center of gravity for computer applications shifts from the host computer to the workstation. This represents a major change in the paradigm for the delivery of computer applications. The computational power available on these workstations is growing at a pace far faster than the growth in traditional host computer capabilities. Moreover, each workstation's power is dedicated to a single user. Applications can be written that require a highly responsive system (such as GUI's), since the designers need not worry that computational power may not be available due to resource sharing.

Intelligent workstations are required to supply a GUI. Where a host computer is to supply the logic of an application, the application is split into a "front-end" and a "backend". The front-end is provided by the workstation and, at a minimum, supplies the GUI. The front-end may also contain some or all of the logic of the application. The backend can provide data access, application logic, and computations. This split of an application is commonly referred to as the client-server architecture. The back-end computers are thought of as "servers" supplying computations or data on demand of the front-end client. The figure below shows some of the different ways applications might be split.

\section{Client-Server Architectures}

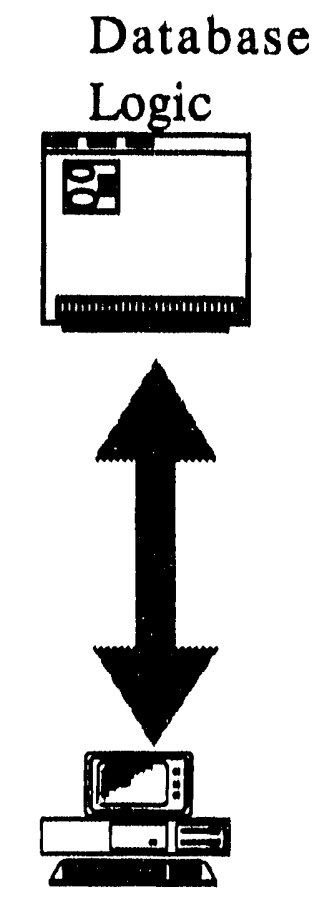

User Interface
Database
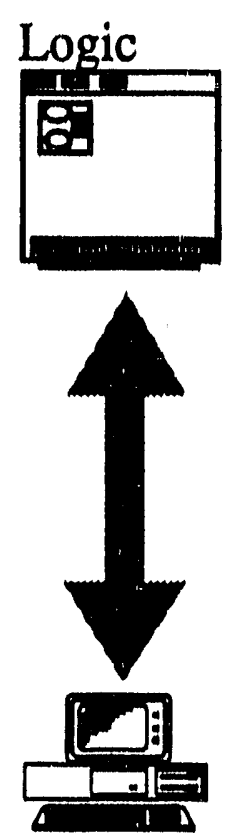

User Interface
Logic

\section{Database}
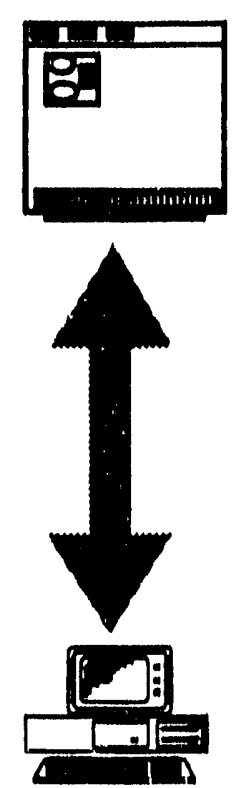

User Interface Logic

\section{Figure 7}




\section{Strategy}

A7 Users will be provided with tools to enhance their ability to generate their own database reports and queries.

\section{Key Features}

Ad hoc query tools using graphical user interfaces.

Reduction in routine printed reports.

Freeing software developers from developing customized reports.

Ability to integrate database queries into workstation analysis tools.

\section{Goals Supported}

I. SRS computing rescurces must be more effectively utilized.

II. Applications and computer systems must be easier to use.

III. The development of applications must be efficient.

VI. End users must be able to know what data is available and have ready access to it.

\section{Challenges}

Data security and integrity concerns.

Forecasting host computer resource use.

Training of end users. 


\section{Description}

The personal computer revolution centered upon putting in the hands of end users (or knowledge workers) computational and data processing tools that allowed them to work directly with their own data. These tools, in general, are more flexible and easy to use than the corresponding programs available on time-shared computers. Moreover, the user was in total control of his or her environment.

However, the down side of using personal computers in a corporate environment is that much of the data that users require to do their jobs reside in corporate "mainframes". What is now required is a joining together of the power and ease of use of the personal computer with corporate data. This will be accomplished by building a communications network that not only physically ties the two together but also supports (with appropriate security features) the extracting of corporate data from mainframe databases and transforming this data to a format that can be used by common personal computer tools.

With appropriate data extraction and analysis tools available to the end user the requirement for mainframe programmers to develop custom reports diminishes. Ad hoc data inquiries by end users also provide more timely snapshots of corporate data and provide the capabilities for more effective analyses of data. If coupled with workstationbased office information systems, the whole process of data inquiry, analysis, and dissemination of the analysis can be end user-generated with the time required to complete the entire process greatly reduced.

Achievement of this environment rests on the selection of appropriate "open" standards for database management systems, network protocols, and workstation tools. Users must also have access to a data repository to direct them to available data. 


\section{References}

1. AED Information Systems: An Integrated Plan. Information Systems Analysis Team, 1985.

2. Government Open Systems Interconnection Profile (GOSIP). FIPS Pub 146, U.S. Department of Commerce, 1988

3. The Benefits of the Graphical User Interface, A Report on New Primary Research. Temple, Barker, and Sloan, Inc., 1990. 


\section{Apondix A}

\section{Summary of Strategies}

\section{Standands}

S1 A site-wide standards infrastructure will be established.

S2 Where applicable, site standards will conform to international and national standards as well as government regulations and DOE orders.

\section{Data}

D1 Data will be managed as a site resource.

D2 A relational database product will be implemented on the mainframe tier and the workgroup tier. Connectivity will be established between the products.

D3 A site-wide data repository will be implemented for the storage of information describing site data and databases.

D4 Referential integrity will be imposed by the RDBMS wherever possible, instead of by individual applications.

D5 A site-wide data administration organization will be established.

\section{Platforms}

P1 System acquisitions will be based on site standards and guidelines.

P2 Systems and workstations will be closely integrated into the site computer network with supporting services.

P3 Robust mission-essential computing and process control facilities will be maintained.

P4 There will be a hierarchy of shared data storage capabilities provided for the site.

\section{Communications}

C1 Data communications services and products will be based on standards.

C2 The site network will be composed of robust LAN trunks and inter-area (facility) links.

C3 Workstations and host computers will connect directly to the site LAN. 


\section{Applications}

A1 The development and purchase of software will be based on site standards.

A2 Off-the-shelf software will be used to the maximum extent possible.

A3 CASE and other computer-based tools will be used to automate the building of applications.

A4 Applications will become modular.

A5 Applications will conform to site standards for common graphical user interfaces.

A6 The capabilities of the intelligent workstation will be a key component of the architecture.

A7 Users will be provided with tools to enhance their ability to generate their own database reports and queries. 


\section{Apsendix B}

\section{Goals vs. Strategies}

The following table shows the relationship of the strategies developed in Section 2 to the goals defined in Section 1.

\begin{tabular}{|c|c|c|c|c|c|c|c|c|}
\hline & I & II & III & IV & $\bar{V}$ & $\overline{\mathbf{V}}$ & VII & ViI \\
\hline S1 & $\sqrt{ }$ & & & $\sqrt{ }$ & & & & \\
\hline $\mathbf{9}$ & $\sqrt{ }$ & & & $\sqrt{ }$ & & & & \\
\hline D1 & 1 & & & & & $\sqrt{ }$ & $\checkmark$ & \\
\hline $\mathbf{D 2}$ & $\checkmark$ & $\sqrt{ }$ & & $\sqrt{ }$ & $\sqrt{ }$ & $\sqrt{ }$ & $\sqrt{ }$ & \\
\hline $\mathbf{D B}$ & & & $\sqrt{ }$ & $\sqrt{ }$ & $\sqrt{ }$ & $\checkmark$ & & \\
\hline DA & 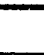 & & & & & & $\sqrt{ }$ & \\
\hline$\overline{D 5}$ & $\sqrt{ }$ & & & & $\sqrt{ }$ & & $\sqrt{ }$ & \\
\hline P1 & $\sqrt{ }$ & & $\checkmark$ & $\sqrt{ }$ & & & & \\
\hline $\mathbf{P 2}$ & $\sqrt{2}$ & $\sqrt{ }$ & $\checkmark$ & $\checkmark$ & & & & \\
\hline$\overline{\mathbf{P 3}}$ & & & & & $\sqrt{ }$ & & & $\sqrt{ }$ \\
\hline $\mathbf{P 4}$ & 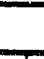 & & & 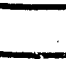 & $\sqrt{ }$ & & & $\sqrt{ }$ \\
\hline $\mathbf{C 1}$ & $\sqrt{ }$ & & & $\sqrt{ }$ & & & & $\sqrt{ }$ \\
\hline $\mathbf{C 2}$ & $\sqrt{ }$ & & & $\sqrt{ }$ & & & & $\sqrt{ }$ \\
\hline C) & $\checkmark$ & $\sqrt{ }$ & $\checkmark$ & $\sqrt{ }$ & & & & $\sqrt{ }$ \\
\hline A1 & $\sqrt{ }$ & $\sqrt{ }$ & & $\sqrt{ }$ & & & & \\
\hline$\overline{A 2}$ & $\sqrt{ }$ & & $\sqrt{ }$ & & & & & \\
\hline A3 & & & $\sqrt{ }$ & & & & & \\
\hline$\overline{A M}$ & $\checkmark$ & & $\checkmark$ & $\sqrt{ }$ & $\sqrt{ }$ & & $\sqrt{ }$ & \\
\hline A5 & $\sqrt{ }$ & $\sqrt{ }$ & & $\sqrt{ }$ & & & & \\
\hline $\mathbf{A B}$ & $\checkmark$ & $\sqrt{ }$ & & $\sqrt{ }$ & & & & \\
\hline A7 & $\sqrt{ }$ & $\sqrt{ }$ & $\sqrt{ }$ & & & $\sqrt{ }$ & & \\
\hline
\end{tabular}




\section{Ampendix C}

\section{Acronyms}

\begin{tabular}{|c|c|}
\hline $\begin{array}{l}\text { ADP } \\
\text { API }\end{array}$ & $\begin{array}{l}\text { Automated Data Processing } \\
\text { Applications Program Interface }\end{array}$ \\
\hline BSRI & Bechtel Savannah River Incorporated \\
\hline CASE & Computer Aided Software Engineering \\
\hline CAST & Computing Architecture and Standards Team \\
\hline CCF & Central Computer Facility \\
\hline CUA & Common User Access (IBM graphical user interface standard) \\
\hline DB2 & IBM's primary relational database management system \\
\hline DBMS & Database Management System \\
\hline DCE & Distributed Computing Environment (OSF) \\
\hline $\mathrm{DEC}$ & Digital Equipment Corporation \\
\hline $\mathrm{DOE}$ & Department of Energy \\
\hline ESCC & Engineering and Scientific Computing Committee \\
\hline Ethernet & local area network technology (DEC/XEROX/INTEL) \\
\hline FDDI & Fiber Distributed Data Interface \\
\hline GIU & Graphical User Interface (window-style interface to computer) \\
\hline GOSIP & Government Open Systems Interface Profile \\
\hline IBM & International Business Machines \\
\hline IEEE & Institute of Electrical and Electronics Engineers \\
\hline IEEE 802.3 & LAN standard for Ethernet \\
\hline IEEE 802.4 & LAN standard for MAP \\
\hline IEEE 802.5 & LAN standard for Token Ring \\
\hline $\mathrm{I} / \mathrm{O}$ & Input/Output \\
\hline IRM & Information Resource Management \\
\hline ISO & International Standards Organization \\
\hline LAN & Local Area Network \\
\hline MAP & Manufacturing Automation Protocol \\
\hline $\mathrm{mbps}$ & Mega bits per second (one million bits per second transmission rate) \\
\hline MOTIF & Graphical User Interface defined by OSF for X-windows \\
\hline MS-DOS & Disk Operating System for IBM-PC (Microsoft) \\
\hline MVS & Multiple Virtual Storage (IBM mainframe operating system) \\
\hline ODIF & Office Document Interchange Format \\
\hline OODB & Object Oriented DataBase \\
\hline OOPS & Object Oriented Programming Syetem \\
\hline $\mathrm{OS} / 2$ & Operating System 2 for IBM PS/2 \\
\hline OSI & Open Systems Interconnection (International Standards Organization) \\
\hline OSF & Open Systems Foundation \\
\hline & Personal Computer (e.g. Mac, IBM) \\
\hline PC/AT & IBM's 1985 PC offering \\
\hline $\mathrm{PC} / \mathrm{XT}$ & IBM's $1982 \mathrm{PC}$ offering \\
\hline PDEC & Process Digital Equipment Committee \\
\hline RSI2 & IBM Personal Systems /2 personal computer \\
\hline SIX & Portable Operating System Interface (IEEE) \\
\hline & Remote Data Access \\
\hline IS & Relational DataBase Management System \\
\hline & Remote Procedure Call \\
\hline
\end{tabular}


SNA Systems Network Architecture (IBM)

SNMP Simple Network Management Protocol

SQL

SRS

Structured Query Language

TCP/IP

UCNI

UNIX

VAX

VMS

VT100

Savannah River Site

Transmission Control Protocol / Internet Protocol

WSRC

Unclassified Controlled Nuclear Information

Portable operating system (TM of AT\&T)

Primary DEC computer family

Virtual Memory System (VAX operating system)

$\mathrm{X} /$ Open Consortium promoting interoperability

A DEC dumb terminal

Westinghouse Savannah River Company

$\mathrm{X}$-window Standard for device-independent text and image network terminals 


\section{Aproendix D}

\section{Information Worker Scenario}

[The following is a scenario of the working life of an SRS information worker using the capabilities which are promised in this Architecture document.]

Hank turns on his workstation and clicks on the icon which logs him onto the network (the only logon he needs to make for the day). A message displays in a "pop-up" window indicating that a new version of his word processor is now available and has been loaded on the file server Hank is using. He is also informed that there are several new bulletin board items which he has not read. The titles, "C-area road closure", "TNX deer hunt", "Safety Alert", and "Car pool parking", are displayed automatically. Hank "clicks on", or selects, the one labeled "Car pool parking" and reads it. He also clicks on the title for "Safety Alert" and reads it, since it's flagged that the item is mandatory reading. (The systems will enforce the requirement if Hank tries to ignore it.) He then clicks on the delete button for the other items. He'll be on vacation starting tomorrow, so the road closure and deer hunt won't affect him

When he logged on, Hank was also informed that he has new electronic mail, there are events scheduled for the day, and that his monthly report is due. Hank decides not to read his mail right now, he wants to check his calendar first. Hank didn't like using electronic calendars until the new version was installed that looks just like his old daytimer. Checking, he notes that the group staff meeting for today has been cancelled, but that his meeting with Mark is still on. Clicking on the meeting time, he's given a full description of the meeting, including attendees and location. Hank notes that Mark has invited two additional people since the last time he checked. Hank cliciss on the room name to display an area map with the building highlighted, he then checks a more detailed building map to see where the room is. He thinks that this is a handy feature of the system, since he's never been to TNX before.

Hank next checks his mail by clicking on the mailbox icon on his desktop. He has 3 new messages. Clicking on the first, he sees that it's a note from Joe about the new pump they ordered. Clicking on the second, he finds a note from Sally, someone at Los Alamos that he's working with on process control design changes. Hank decides to forward a copy to the local vendor representative for the control system that Sally is discussing (since he wasn't copied on the message) after adding a few explanatory words.

Hank wonders if there are any controllers of the type being used in the control system in stores or used elsewhere at the site, so he decides to check. He clicks on the data access tool on his desktop and is presented a list of major subject areas. Hank clicks on "Equipment" and sees a list of attributes; he clicks on manufacturer, and asks for a list of values. Seeing the name of the vendor in question, he clicks on the name to select the vendor. He next clicks on the part number field and again asks for a list of values, which results in several parts, including the one he's looking for. "Great!", he thinks, "now all I have to do is see where they are..." Hank asks for additional detail by clicking on the location and status attributes, and sees that there are two controllers in use in 221-F. Wondering if any are in stores, he asks to see a diagram of relationships associated with equipment. He sees that spare parts are related to equipment so he 
requests a search of spares for the given controller part number. The system shows that there are two in stock.

Hank leaves the data access window on his screen, but clicks on the application tool on his desktop. He scrolls through the available applications until he finds "Spare Part Order" and clicks on that entry. A new window appears with a stores order form. Hank fills in the necessary part caption information (copying it from the data access window) and clicks "OK". The spare controller he just ordered should be shipped by stores today.

Hank is also needs to check on the design schematic of the process control system where the controller is to be used. He brings up a window on his workstation for viewing drawings in the site Computer Aided Design (CAD) drawing repository. Since he wants to sketch in some alternative configurations, Hank files a copy of the drawing on his workstation's file system. Hank plans to use his personal computer CAD software package later in the day to sketch in proposed changes to the system.

Ordering the controller reminded Hank that he wants to check on the status of several purchase orders that are in progress, so he selects the "Purchase Requisition. Inquiry" application. A window displays a list of his outstanding requisitions (since the system already knew who he was). Hank clicks on one of his requisitions, and a detail status displays in another pop-up window on his screen. He pulls down the application main menu and closes all the open windows to tidy up his screen. It's time to finish reading his mail.

Hank's third message was from Mark, emphasizing items that need to be included in this month's monthly report. Hank decides it's time to get it over with, so he starts the word processing application and begins writing his section of the report. He decides that he wants to include some comments on the excellent, QIP results that his group has this month, so he clicks on the EIS tool ir. his application folder. A window automatically appears from which he selects QIP. A menu displays which makes available the QIP results for Hank's division, department, section, and group. He selects his group, scrolls through the available display, and selects the graph he is interested in. When it displays, he uses electronic "cut and paste" operations to copy it into his word processing document, after adding an annotation explaining the dramatic improvement. Hank next opens his group's shared monthly report folder and brings in excerpts from the group members' reports. He finishes his report, archives a copy by dragging it to his archive folder (for storage in the the central network archives), and puts the document onto the server in a folder where Mark can get to it.

"Time for a Diet Coke," Hank thinks, "it's already 9:00". 


\section{Apoendix E}

\section{Management Scenario}

[The following is a scenario of the working life of an SRS senior manager using the capabilities which are promised in this Architecture document.]

Jim comes into his office to find his workstation already logged on to the SRS Executive Information System (EIS) at the window that summarize the operating/safety events since the previous day. These events have been extracted from the morning reports Safeguards and Security, Operation, and Environmental.

Jim interrogates each of these areas to get a quick review of the operating status of the site and issues a set of questions and requests to responsible senior management about specific issues. Jim next calls up the bulletin board window listing all major events happening at SRS today and pulls up his calender to see if he should/can change his calender to attend any of these events.

Jim has been thinking about the GRH budget situation and site manpower and realizes that there could be a shift in resources required. To see what the situation is, Jim calls up windows giving the manpower and expenditures for all projects above $\$ 5,000,000$, the manpower requirements and costs for each large operating facility, and does an "What If" analysis on the question: "What should we do to absorb a $10 \%$ budget cut". Realizing that the computer does not contain enough in-depth model information, he generates a series of questions and a list of candidate actions that should be analyzed. He sends these questions and lists to his senior staff electronically, along with voice annotations to each in preparation for tomorrow's staff meeting.

Jim has a few minutes before he needs to go on an audit of K-Reactor and pulls up a window allowing him to find the office location of Sam, the person who will lead the audit. He pulls up some of Sam's personnel background information, including a picture, and leaves for the audit.

Upon returning, Jim accesses a window into the Commitment Management System relative to SRS Environmental Commitments in preparation for a meeting with DHEC and DOE tomorrow afternoon. After reviewing these commitments, Jim sends a list of questions to Jack in Environmental Protection that must be answered by this afternoon, and he sets an autodial conference call to Jack for 3:00 p.m.

Jim says it's now time for the Expanded Staff Meeting and departs for 773-A Conference Room. (This could be done via a video-based conference, but the personal touch will always be in vogue).

After lunch, Jim accesses the response to his question about an earlier operational incident. The reply says that a video has been prepared showing the incident scene. Jim brings this video up on his workstation to get a first hand view hefore the Policy Review Committee meeting. At Policy Review, all incidents are reviewed via video tape 
through a window projected on a large screen, with each incident supported by data and text.

Jim returns to his office at 3:00 p.m. to receive the Conference Call from Jack relative to the Environmental Meeting tomorrow. Jack has the situation well in hand, and Jim pulls up a window on the current status of the SCUREF project to review any issues identified via his EIS system. Noting that all is well, Jim pulls up a window giving a summary of project status compared to plan, beginning with the largest projects. He sees that a project in X Area is 20\% behind schedule and requests an overview for next week on the relevant issues and a plan to get back on schedule.

Jim next pulls up his agenda for the Weekly Meeting with DOE to review his thoughts on how to present the issues to give consensus.

It's now 5:00, and Jim leaves to attend a function in Augusta. 


\section{Anoxumdix F \\ Application Developer Scenario}

[The following is a scenario of the working life of an SRS software applications developer using the capabilities which are promised in this Architecture document.]

Headquarters, Department of Energy (DOE) has ordered the consolidation of several production facilities and selected the Savannah River Site (SRS) as it's facility for the production of Fuel W. Joe, a Westinghouse Savannah River Company (WSRC) information analyst assigned to Information Resources Management (IRM) is given the responsibility for supporting the information systems requirement for Fuel W.

Joe hosts a Joint Application Development (JAD) session to define the information requirements of Fuel W. In this series of meetings, the WSRC organizations responsible for producing the fuel meet with information analysts from IRM to define the information requirements in terms of process and data flows, business rules, and data entity relationships. They are creating an Enterprise Model for the Fuel W process. Joe records the information using a CASE tool on his portable computer. At the conclusion of the the final JAD session, Joe is asked to produce a preliminary analysis document and send copies to each JAD attendee as well as the site's Data Administration group and IRM development managers.

Returning to his office, Joe transfers the enterprise model to his workstation's CASE library. Opening up a development window on his workstation, Joe clicks on the icon for CASE causing a window to pop up and display icons for the models assigned to Joe's development team. Clicking on the Fuel W icon, Joe creates an analysis report and uses electronic mail to route the report to interested parties.

After incorporating everyone comments, Joe's next task is to develop a prototype design based on the rules, flows and objects identified during the analysis phase. The prototype will run on his workstation in a windows environment that emulates the production operating system and databases.

Each team member is assigned portions of the design. Tom is responsible for data, David and Bill for prototyping individual functions. Tom, as the team's data analyst is responsible working with the site's Data Administration (DA) group and their data repository. Tom's initial task is to populate the application model with meta data extracted from the site's data repository.

Tom accesses the repository, seeking the business rules along with the process and data flows associated with the objects identified through the JAD session.

The repository security system acknowledges that Tom has authorization to acces and retrieve information but does not have authorization to modify the repository. 
Tom populates the model with the meta data contained in the repository. However, for the objects not defined to the repository, Tom must consult with DA requesting their approval for the new objects. Bill, David, and Joe validate the populated model against their understanding of the requirements. Joe detects several questionable data flows, and potential modifications to exiting flows and processing rules. He asks Tom to coordinate the modifications with DA and other IRM development teams affected by the changes.

Processing rules contain the data validation criteria for olbjects stored in a databese and which are automatically invoked by DBMS when objects are modified. Rules processing eliminates the practice of hardcoding validation criteria into applications.

Additionally, Joe asks Tom to generate the Relational Data Model, the final step in the analysis phase. Tom use his Relational Model CASE tool to generate the Fuel W Relational Data Model.

Bill, David and Joe construct prototype screens, reports, modules and structure charts using workstation-based software. Inherent with the screen and roport generators are the rules for site standards including graphical user interfaces (GUI).

Joe loads the completed prototype to a "test" file server and sends an electronic message alerting IRM development teams, Data Administration, and selected user in the Operating Department that the prototype design for Fuel $\mathrm{W}$ is available for review and comment.

The 'test' file server is accessible to all participating development teams and review groups.

For the necessary software creation phase Joe elects a combination of coding solutions: CASE-generated code for the data collection and maintenance functions and Fourth Generation Language (4GL) report writing for query access and data reporting. Tom coordinates with Data Administration to insure that the test repository is updated to contain the modified processing rules, and Database Administration (DBA) generates the relational databases. Joe together Computer \& Telecommunications Management (CTM) and Fuel W users determine the appropriate platform(s) for execution of the application. They conclude that the data entry portion should be run on a workgroup server while the remainder should to run on the CCF's IBM mainframe. Satisfied that the design requirements supporting code generations are as complete as possible, Joe give his team the go-ahead.

Bill and David, at their workstations, access the CASE, Construction Generator. The construction generator requests information as to operating platform and database. When this information is supplied the Construction Generator creates SQL-compliant code. However, both Bill and David know that the initial code is generic, and requires tailoring to site specific requirements. They supplement the generated code with precoded routines pulled from Supplemental Reusable Code library. Meanwhile, Tom codes the query and reporting functions using the site's standard query and report writing tool. 
Coding completed, the development team unit tests the application using files contained on their local file server. In addition to standard systems testing, Joe tests the referential linkages with several distributed subject-oriented data bases located across hardware and operating platforms. With this testing successful, Joe requests the Operating Departments and IRM development groups test the Fuel W system.

When testing is completed, DA generates the files and incorporates the model into the site repository, CTM accepts and loads the code from Joe's workstation to the production file server and alters customer profiles, adding the Fuels $W$ icon to user interfaces screens. 


\section{AposendixG}

\section{Advanced Technologies}

The planning period for this architecture has been established at eight years. There are several emerging advanced computing technologies which may mature within that thme frame. If so, these will have an impact on the SRS computing architecture, although there is too much uncertainty about how these technologies will evolve to include them as integral parts of the architecture today. CAST will continue to monitor the state of their implementations, and will, if necessary, modify future architecture statements to incorporate new technologies which provide capabilities which are needed to fulfill the site mission or which replace older technologies in more cost effective ways. The Advanced Technology and Architecture (ATA) Section of IRM will aiso conduct pilot studies on new technologies to investigate their possible impact on the site computing architecture.

These technologies are described in the paragraphs below.

\section{Neural Networks}

A neural network is a new kind of computing system that is inspired by, but does not rectually mimic, a human brain. It shows great potential for pattern recognition, control system, signal processing, and expert system applications. A neural network is different from a traditional digital computer on three counts: first, it has no central processing system but is instead constructed with neurodes, small processing elements that are highly interconnected; second, it has no separate memory for data storage; and third, the neuroiles respond to a particular pattern of input signals by generating an output signal appropriate to the total simulation received.

In the future, neural networks may be used to solve site problems not easily tractable by conventional computing. These problems include analysis of reactor data (for example, pattern recognition, seismic data analysis, and image analysis of $x$-rays and sonograms of reactor components), robotics, and information retrieval systems.

\section{Multimedia}

Multimedia is a catch-all term to describe the use of computing to integrate information delivery at the workstation. Information can take many forms, such as combinations of text, computer database or file access, graphics files, images, full motion video, or voice output. There are many custom multimedia systems available for purchase today. The major question for incorporating multimedia capabilities in the architecture is determining when the economies of the various supporting technologies are such that mainstream information systems can incorporate multimedia capabilities. Early implementations of multimedia will be seen at the site in computer-aided training systems, executive information systems, and process monituring systems. 


\section{Advanced Input Devices}

Integrating voice input capabilities at the workstation has been promised by the industry for several years. As in the case of multimedia applications, use of informations systems that integrate voice input is largely a matter of economics. There are systems available today that are speaker-independent with a vocabulary of several hundred words. These will prove useful in certain applications long before the state of the technology makes it economic as well as beneficial that the average information worker have access to this capability.

Another technology that could emerge in general applications is the use of a computer stylus to allow handwritten input to the computer. Such input could be used for verification of signatures, but the technology also promises optical character recognition of handwriting to allow conversion of handwritten input to "machine-readable" text.

\section{Artificial Reality}

Artificial reality, also called environmental simulation, is a term used to express the combination of advanced sensory input capabilities with highly precise computer simulation models to be able to build simulations of such detail and quality as to be indistinguishable from the world being simulated. This technology is being tested today by the military for advenced flight simulators, and a 1980's version of the technology exists in the site's Reactor Simulator.

\section{Massively Parallel Computing}

The concept of massively parallel computing is to reduce problems to many small components and to solve these components in parallel with a processor complex consisting of many tightly coupled processors. In this manner, individual computers need not continue to ? et faster in order to solve complex problems faster. This technology is in use today in specialized applications that model well into parallel components ( for example, reactor simulations, environmental modeling, and graphics image processing) and in database management systems, which use many processors to share the workload generated by concurrent database access requests. It is expected that massively parallel computing will tend to obsolete and replace vector processing used in today's supercomputers.

\section{Database Machines}

Database machines are dedicated to providing a database management system "server" to clients throughout an enterprise network. These machines have spacialized hardware that optimizes the computer for the task of relational queries. There is no question that the site will be going to database "servers"; the major question is whether it is more economical in the long run to use specialized hardware or to use general purpose computers with specialized software (that is, datsbase server code). 

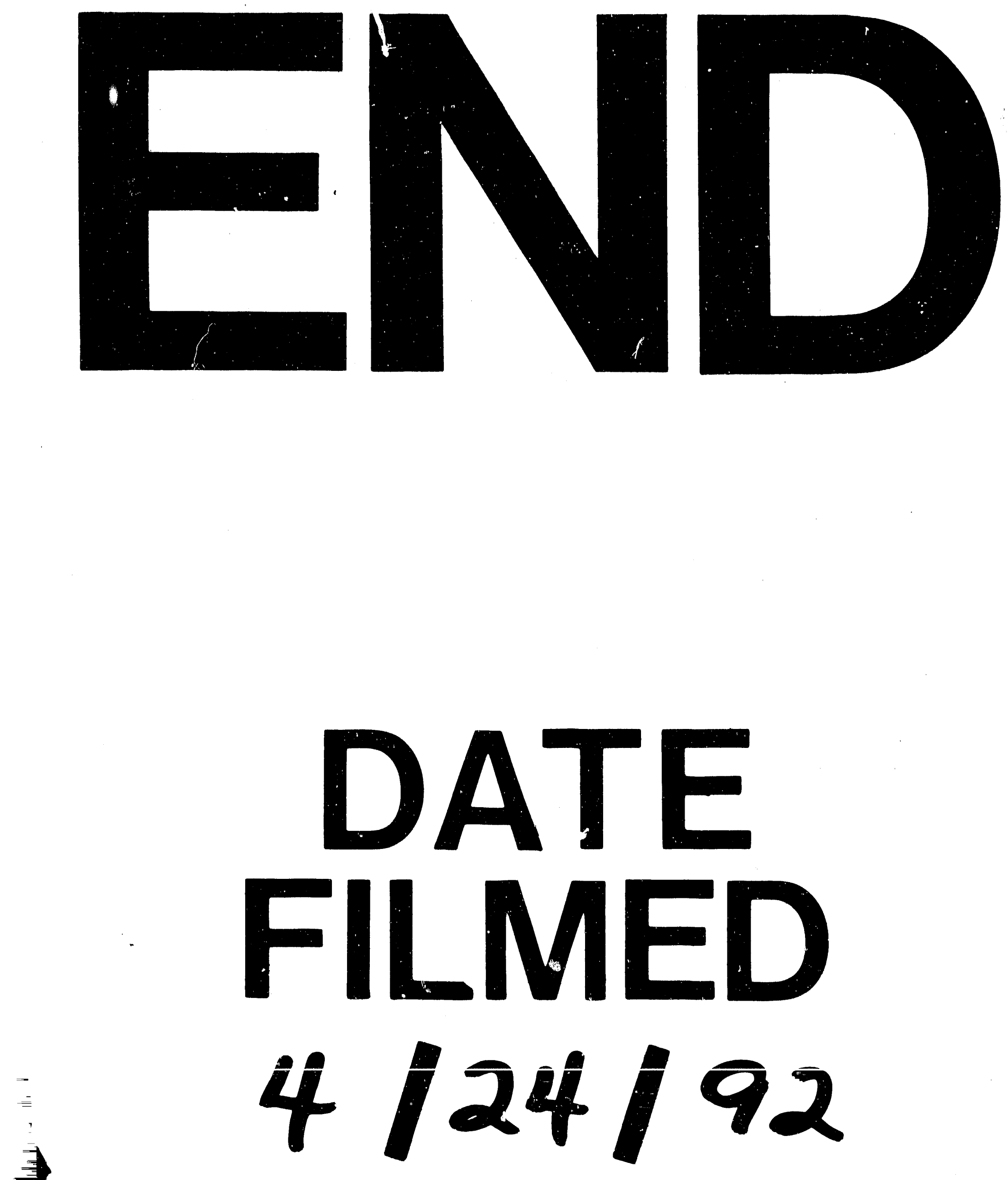
